\title{
Cross-ratio identities and higher-order poles of CHY-integrand
}

\author{
Carlos Cardona, ${ }^{a}$ Bo Feng, ${ }^{b, c}$ Humberto Gomez ${ }^{d, e}$ and Rijun Huang ${ }^{b, 1}$ \\ ${ }^{a}$ Physics Division, National Center for Theoretical Sciences, National Tsing-Hua University, \\ No.101, section 2, Kuang-Fu Road, Hsinchu, Taiwan 30013, Republic of China \\ ${ }^{b}$ Zhejiang Institute of Modern Physics, Department of Physics, Zhejiang University, \\ No.38, Zheda Road, Hangzhou, 310027, P.R. China \\ ${ }^{c}$ Center of Mathematical Science, Zhejiang University, \\ No.38, Zheda Road, Hangzhou, 310027, P.R. China \\ ${ }^{d}$ Instituto de Fisica - Universidade de São Paulo, \\ Caixa Postal 66318, 05315-970 São Paulo, SP, Brazil \\ ${ }^{e}$ Facultad de Ciencias Basicas, Universidad Santiago de Cali, \\ Calle $5 N^{\circ}$ 62-00 Barrio Pampalinda, Cali, Valle, Colombia \\ E-mail: carlosandres@mx.nthu.edu.tw, fengbo@zju.edu.cn, \\ humgomzu@gmail.com, huang@nbi.dk
}

Abstract: The evaluation of generic Cachazo-He-Yuan(CHY)-integrands is a big challenge and efficient computational methods are in demand for practical evaluation. In this paper, we propose a systematic decomposition algorithm by using cross-ratio identities, which provides an analytic and easy to implement method for the evaluation of any CHYintegrand. This algorithm aims to decompose a given CHY-integrand containing higherorder poles as a linear combination of CHY-integrands with only simple poles in a finite number of steps, which ultimately can be trivially evaluated by integration rules of simple poles. To make the method even more efficient for CHY-integrands with large number of particles and complicated higher-order pole structures, we combine the $\Lambda$-algorithm and the cross-ratio identities, and as a by-product it provides us a way to deal with CHY-integrands where the $\Lambda$-algorithm was not applicable in its original formulation.

KEYwords: Scattering Amplitudes, Differential and Algebraic Geometry

ARXIV EPRINT: 1606.00670

\footnotetext{
${ }^{1}$ Corresponding author.
} 


\section{Contents}

1 Introduction 1

2 The cross-ratio identities 4

$\begin{array}{lll}3 & \text { A systematic decomposition algorithm } & 11\end{array}$

$\begin{array}{ll}3.1 \text { The algorithm } & 11\end{array}$

$\begin{array}{lll}3.2 & \text { An illustrative example } & 13\end{array}$

4 The $\Lambda$-algorithm and the cross-ratio identities $\quad 15$

$\begin{array}{lll}4.1 \text { Some notations } & 15\end{array}$

$\begin{array}{ll}4.2 & \text { A simple example } \\ & 16\end{array}$

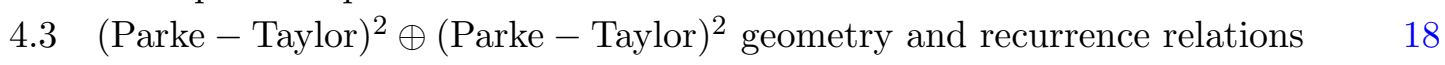

5 Conclusion $\quad 22$

A A practical algorithm to determine the \pm sign in integration rules $\quad 24$

B The identities from monodromy relation and cross-ratio identities $\quad 28$

C (Parke - Taylor $) \times($ Parke - Taylor $)$ geometry and its recurrence relation 30

D Recurrence relation for $\mathcal{I}_{3,3}^{\mathbf{P T}^{2} \oplus \mathbf{P T}}{ }^{2}, \mathcal{I}_{4,3}^{\mathbf{P T}^{2} \oplus \mathbf{P T}}{ }^{2}, \mathcal{I}_{5,3}^{\mathbf{P T}^{2} \oplus \mathbf{P T}}{ }^{2}$ and $\mathcal{I}_{6,3}^{\mathbf{P T}^{2} \oplus \mathbf{P T}^{2}} 32$

\section{Introduction}

The $n$-particle scattering amplitude in arbitrary dimension can be described by CachazoHe-Yuan (CHY) formulation [1-5] as

$$
\begin{aligned}
\mathcal{A}_{n}^{\text {tree }} & =\int\left(\frac{\prod_{a=1}^{n} d z_{a}}{\operatorname{vol~SL}(2, \mathbb{C})}\right)\left(\prod^{\prime} \delta\left(\mathcal{E}_{a}\right)\right) \mathcal{I}(z) \\
& =\int\left(z_{r s} z_{s t} z_{t r} \prod_{a \in\{1,2, \ldots, n\} \backslash\{r, s, t\}} d z_{a}\right)\left(z_{i j} z_{j k} z_{k i} \prod_{a \in\{1,2, \ldots, n\} \backslash\{i, j, k\}} \delta\left(\mathcal{E}_{a}\right)\right) \mathcal{I}(z),
\end{aligned}
$$

respecting Möbius $\mathrm{SL}(2, \mathbb{C})$ invariance. The scattering equations $\mathcal{E}_{a}$ 's form an algebraic system of $n$ rational functions in $n$ complex variables $z_{a}, a=1,2, \ldots, n$ as

$$
0=\mathcal{E}_{a}=\sum_{b \in\{1,2, \ldots, n\} \backslash\{a\}} \frac{s_{a b}}{z_{a}-z_{b}} \text { for } a=1,2, \ldots, n,
$$

where $\{1,2, \ldots, n\} \backslash\{a\}$ denotes a set of $\{1,2, \ldots, n\}$ extracting the element $a$. Instead of (1.3), it has been shown in [6] that an equivalent polynomial form of scattering equations 
exists, with its geometric structure investigated in [7], makes the evaluation of (1.2) wellsuited in the algebraic geometry context. The Möbius invariance implies that among the $n$ distinct punctured points $z_{a}, a=1,2, \ldots, n$ in Riemann sphere, any three of them, say $z_{a}, a \in\{r, s, t\}$, can be fixed to particular locations, conventionally chosen as $z_{r}=\infty, z_{s}=$ $1, z_{t}=0$, such that the $n$-dimensional integration module the volume of $\operatorname{SL}(2, \mathbb{C})$ in $(1.1)$ can be written as the one in the first parenthesis of (1.2), which is a $(n-3)$-dimensional integration. The Möbius invariance also implies that only $(n-3)$ scattering equations are linearly independent, and it forces us to write the $\prod^{\prime} \delta\left(\mathcal{E}_{a}\right)$ in (1.1) as the one in the second parenthesis of $(1.2)$, to ensure that $\prod^{\prime} \delta\left(\mathcal{E}_{a}\right)$ is independent of removing any three scattering equations $\mathcal{E}_{a}, a \in\{i, j, k\}$, leaving only $(n-3)$ linearly independent delta functions. Hence formula $(1.2)$ is in fact a $(n-3)$-dimensional integration constrained by $(n-3)$ delta functions, allowing a representation

$$
\mathcal{A}_{n}^{\text {tree }}=\sum_{z \in \text { solutions }} \frac{z_{i j} z_{j k} z_{k i} z_{r s} z_{s t} z_{t r}}{\text { Jacobian }} \mathcal{I}(z),
$$

on the $(n-3)$ ! solutions of scattering equations, where

Jacobian $=\operatorname{det}\left[\frac{\partial \mathcal{E}_{a}}{\partial z_{b}}\right]_{(n-3) \times(n-3)}, \quad$ for $a \in\{1,2, \ldots, n\} \backslash\{i, j, k\}, \quad b \in\{1,2, \ldots, n\} \backslash\{r, s, t\}$,

coming from the evaluation of $(n-3)$ delta functions. The so called $C H Y$-integrand $\mathcal{I}(z)$ is a rational function of $z_{i j} \equiv z_{i}-z_{j}$, external momenta $k_{i}$ 's as well as the polarization vectors $\epsilon_{i}$ 's, whose explicit definition varies with the field theories under consideration, while systematic and compact construction of $\mathcal{I}(z)$ exists for bi-adjacent cubic-scalar, pure Yang-Mills, Gravity theories, NLSM, DBI as well as mixing among them [3-5].

Although conceptually simple and elegant, it is in fact impossible to analytically evaluate (1.2) by (1.4), due to the well-known Abel-Ruffini theorem that there is no algebraic solution to the general polynomial equations of degree five or higher with arbitrary coefficients. There are a few studies on the solutions of scattering equations in four-dimension and at special kinematics [8-11], but not generic. Even in five-point case where analytic solution of scattering equations is available, the $(5-3) !=2$ solutions are radical functions of Mandelstam variables. Only after summing over two solutions we get rational functions as the final simple result. This infers that there must be better evaluation techniques, and it motives various approaches towards the evaluation of (1.2) avoiding the explicit solutions of scattering equations, based on algebraic geometry techniques. In [12], a method is proposed and applied to analytically evaluating all five-point amplitudes, by the well-known Vieta formulae that relates the sum of solutions of a polynomial equations to the coefficient of polynomials. The elimination theory elaborated therein for rewriting multivariate polynomials as an univariate polynomial is further developed in [13-15], to deal with more generic $n$-particle scattering system. In [16], companion matrix method is introduced, which rephrases the computation of summing over $(n-3)$ ! solutions as computing the trace of certain $(n-3) ! \times(n-3)$ ! matrix, and provides an intuitive interpretation that the final analytic result is indeed rational functions. This is later proven to be equivalent to the elimination method $[13,15]$. In [17], Bezoutian matrix method is introduced to evaluate 
the total sum of residues of (1.2) algebraically, without working out individual residues (or solutions of scattering equations). In [18, 19], polynomial reduction techniques are investigated based on the polynomial form [6] of scattering equation. All above algebraic geometry based methods are in principle generalizable to the evaluation of any $n$-particle scattering, however the elimination algorithm as well as the polynomial reduction rewrite the polynomials in a form such that the coefficients are rather involved. Furthermore, a complete computation also depends on the explicit expression of Jacobian (1.5) which could be very complicated for large $n$. These make the analytic computation in practical difficult even for lower-point amplitude. A resulting trouble is that the amplitude computed by these methods is not in the form with manifest physical poles, and simplification of the result takes quite a long time. For example, using the companion matrix method [16], it would be possible to spend hours to produce a six-point amplitude of scalar theory.

In the demand of computational efficiency, many other methods come to rescue. In [20], graph theory knowledge is introduced in the contour integration of (1.2), to expand a generic CHY-integrand into basis of known simple CHY-integrands, named after building blocks. This idea is further developed in [21], resulting into the so called $\Lambda$-algorithm, to recursively cut a generic CHY-integrand into lower-point sub-CHY-integrands until to certain basic building blocks. Although this method is able to deal with off-shell configurations, which have allowed to generalize it to loop-level recently in [22], and despite it can compute CHY-integrands of higher-order poles, it still has some limitations. The cutting procedure for CHY-integrands is general, but when the so called singular configurations appear in sub-CHY-integrands, evaluation becomes difficult. However for many CHYintegrands, the singular configuration is un-avoidable. Another approach is inspired by the string amplitude computation, where combinatorial rules for integration (integration rules) of $(1.2)$ is derived [23, 24]. In [25, 26], auxiliary Feynman-like diagrams are introduced to compute the global residue, quite similar to that of [23, 24, 27], while in [28], Berends-Giele recursion relations are applied to the computation of CHY-integrands which are products of two Parke-Taylor type factors that containing only simple poles. On the other hand, the integration rule is a rather simple and efficient technique. In fact, the actual computation can be carried out without any information of the solutions of scattering equations as well as the detailed expression of Jacobian in CHY-formulation, but only the information of CHY-integrand as a rational function of $z_{i j}$. However, it suffers from a disadvantage that only CHY-integrand with simple poles can be perfectly evaluated, while a generic CHYintegrand from Yang-Mills or gravity theories could have many higher-order poles. There are two intuitive ways of bypassing this disadvantage. The first is to create rules for higherorder poles, which is investigated in [29], but yet a complete set of rules for any types of higher-order poles are required therein before it could be a complete method. The other is to decompose a CHY-integrand of higher-order poles into several CHY-integrands of simple poles by non-trivial identities relating different rational terms of $z_{i j}$. The idea of decomposition is already discussed in [23], where Pfaffian identities are introduced to take on the task. But the power of Pfaffian identities is limited to certain examples with lower-point 
scattering, and no systematic implementation can be elaborated with them. ${ }^{1}$ In a recent paper [30], identities originated from monodromy relations are proposed to systematically decompose a CHY-integrand of higher-order poles into those of simple poles.

In this paper, we propose another kind of identities, namely the cross-ratio identities, to overcome the difficulties towards a systematic and complete evaluation of (1.2) in practice. These identities are applied in two levels. For a CHY-integrand with reasonable number of scattering particles and not so complicated higher-order pole structures, ${ }^{2}$ the cross-ratio identities can be applied directly to decompose a CHY-integrand into terms with only simple poles. While in the situation that a naive decomposition of a CHY-integrand would result in far too much terms that slow the computation, the $\Lambda$-algorithm developed in [21] would then be introduced to split a CHY-integrand into products of several lower-point CHY-integrands which can not be computed by $\Lambda$-algorithm, followed by the application of cross-ratio identities to reformulate the lower-point ones as those that can be computed by integration rules of simple poles or $\Lambda$-algorithm.

This paper is structured as follows. In section 2, we present the construction of crossratio identities and its generic formulation for an arbitrary pole $1 / s$, and demonstrate the decomposition of CHY-integrands of higher-order poles by cross-ratio identities with two examples. in section 3, we propose a systematic algorithm aims to completely decompose any CHY-integrand of higher-order poles within finite steps by cross-ratio identities, and illustrate the algorithm by a highly non-trivial eight-point example. In section 4, we illustrate how the $\Lambda$-algorithm and decomposition algorithm with cross-ratio identities can work together to make an even more efficient computational method. Recurrence relations for a particular type of CHY-integrands are presented as an example to show that for many complicated CHY-integrands with large $n$, iterative cutting procedure can be applied to rewrite them as lower-point sub-CHY-integrands which are easy to compute. Section 5 comes the conclusion, while in appendix A, a practical algorithm is proposed for a complete implementation of integration rules of simple poles. In appendix B, comparison is provided between the identities from monodromy relations [30] and cross-ratio identities, while in appendix C, D, quantities needed for the recurrence relations by $\Lambda$-algorithm are provided. Very brief introduction on integration rules of simple poles can be found in section 3 and appendix A, but we suggest [29] for more detailed explanation. For detailed description of $\Lambda$-algorithm please refer to [21, 22].

\section{The cross-ratio identities}

Following the decomposition idea, the primary problem is to find a better identity rather than Pfaffian identities, which can be applied to CHY-integrands with any pole structures.

\footnotetext{
${ }^{1}$ For even-integer $n$, a Pfaffian identity relates $2(n-3) ! !$ terms, while for odd-integer $n$, it relates $(n-2) ! !$ terms, so the number of terms involved in an identity grows factorially. But a more severe problem is that the Mandelstam variables involved in Pfaffian identity are only $s_{i j}$, and a pole of $\frac{1}{s_{i_{1} i_{2} \cdot i_{m}}}$ with $m>2$ is not obviously exist.

${ }^{2}$ From the practice, this can be detailed as around $n=10$ with about five higher-order poles (double or triple pole), or any number of particles with around two higher-order poles. They can be computed in a reasonably short time.
} 

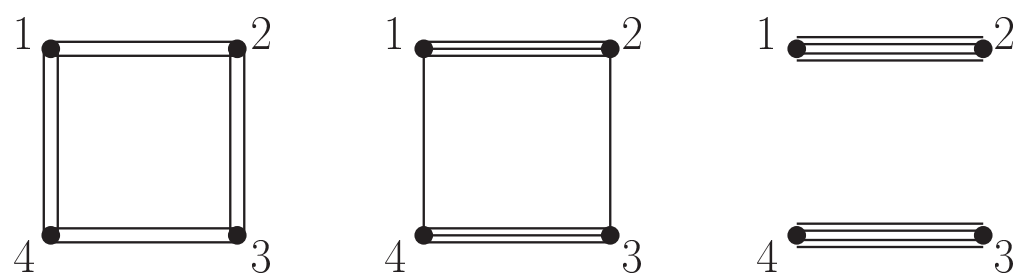

Figure 1. The 4-regular graphs of four-point CHY-integrands with simple, double and triple pole respectively.

Although it is extensively discussed in the literature, let us take, for conceptual inspiration, some four-point CHY-integrands as starting point. Three typical CHY-integrands are given as

$$
\mathcal{I}_{4}^{\mathrm{S}}=\frac{1}{z_{12}^{2} z_{23}^{2} z_{34}^{2} z_{41}^{2}}, \quad \mathcal{I}_{4}^{\mathrm{D}}=\frac{1}{z_{12}^{3} z_{23} z_{34}^{3} z_{41}}, \quad \mathcal{I}_{4}^{\mathrm{T}}=\frac{1}{z_{12}^{4} z_{34}^{4}},
$$

with their 4-regular graphs shown in figure 1.

The explicit results for them are given as

$$
\mathcal{A}\left(\mathcal{I}_{4}^{\mathrm{S}}\right)=-\frac{1}{s_{12}}-\frac{1}{s_{14}}, \quad \mathcal{A}\left(\mathcal{I}_{4}^{\mathrm{D}}\right)=-\frac{s_{13}}{s_{12}^{2}}, \quad \mathcal{A}\left(\mathcal{I}_{4}^{\mathrm{T}}\right)=\frac{s_{13} s_{14}}{s_{12}^{3}} .
$$

The CHY-integrand $\mathcal{I}_{4}^{\mathrm{S}}$ contains only simple poles, and can be readily evaluated by integration rules, while $\mathcal{I}_{4}^{\mathrm{D}}$ and $\mathcal{I}_{4}^{\mathrm{T}}$ contain double pole and triple pole respectively, which makes it impossible to apply integration rules of simple poles. However, a simple factorization of these results

$$
\mathcal{A}\left(\mathcal{I}_{4}^{\mathrm{D}}\right)=-\frac{1}{s_{12}}\left(\frac{s_{13}}{s_{12}}\right), \quad \mathcal{A}\left(\mathcal{I}_{4}^{\mathrm{T}}\right)=\frac{1}{s_{12}^{2}}\left(\frac{s_{13} s_{14}}{s_{12}}\right)
$$

indicates that, if explicit $\frac{1}{s}$ factor can be introduced in the original CHY-integrand to compensate the extra degrees of $s$ from the higher-order poles, then the expressions in the parenthesis are likely to be produced by CHY-integrands of simple poles (dressed with appropriate $s$ ). To explain the above statement, let us start from a scattering equation

$$
\mathcal{E}_{1}=\frac{s_{12}}{z_{12}}+\frac{s_{13}}{z_{13}}+\frac{s_{14}}{z_{14}}=0
$$

and modify it as

$$
\begin{aligned}
0=\frac{z_{12}}{s_{12}} \mathcal{E}_{1} & =1+\frac{s_{13}}{s_{12}} \frac{z_{12}}{z_{13}}+\frac{s_{14}}{s_{12}} \frac{z_{12}}{z_{14}}=1-\frac{s_{12}+s_{14}}{s_{12}} \frac{z_{12}}{z_{13}}+\frac{s_{14}}{s_{12}} \frac{z_{12}}{z_{14}} \\
& =\left(1-\frac{z_{12}}{z_{13}}\right)+\frac{s_{14}}{s_{12}}\left(\frac{z_{12}}{z_{14}}-\frac{z_{12}}{z_{13}}\right)=\frac{z_{23}}{z_{13}}+\frac{s_{14}}{s_{12}} \frac{z_{12} z_{13}}{z_{14} z_{13}} .
\end{aligned}
$$

From the last expression we end up with an identity

$$
1=-\frac{s_{14}}{s_{12}} \frac{z_{12} z_{43}}{z_{14} z_{23}} .
$$

Since the $z_{i j}$ 's in the identity are arranged as cross-ratios, which are invariant under Möbius transformation, we call it the cross-ratio identity. Note that a pole $\frac{1}{s_{12}}$ is apparent in the identity. Multiplying it with $\mathcal{I}_{4}^{\text {D }}$ leads to

$$
\mathcal{I}_{4}^{\mathrm{D}}=\left(\frac{1}{z_{12}^{3} z_{23} z_{34}^{3} z_{41}}\right) \times\left(-\frac{s_{14}}{s_{12}} \frac{z_{12} z_{43}}{z_{14} z_{23}}\right)=-\frac{s_{14}}{s_{12}} \mathcal{I}_{4}^{\mathrm{S}},
$$



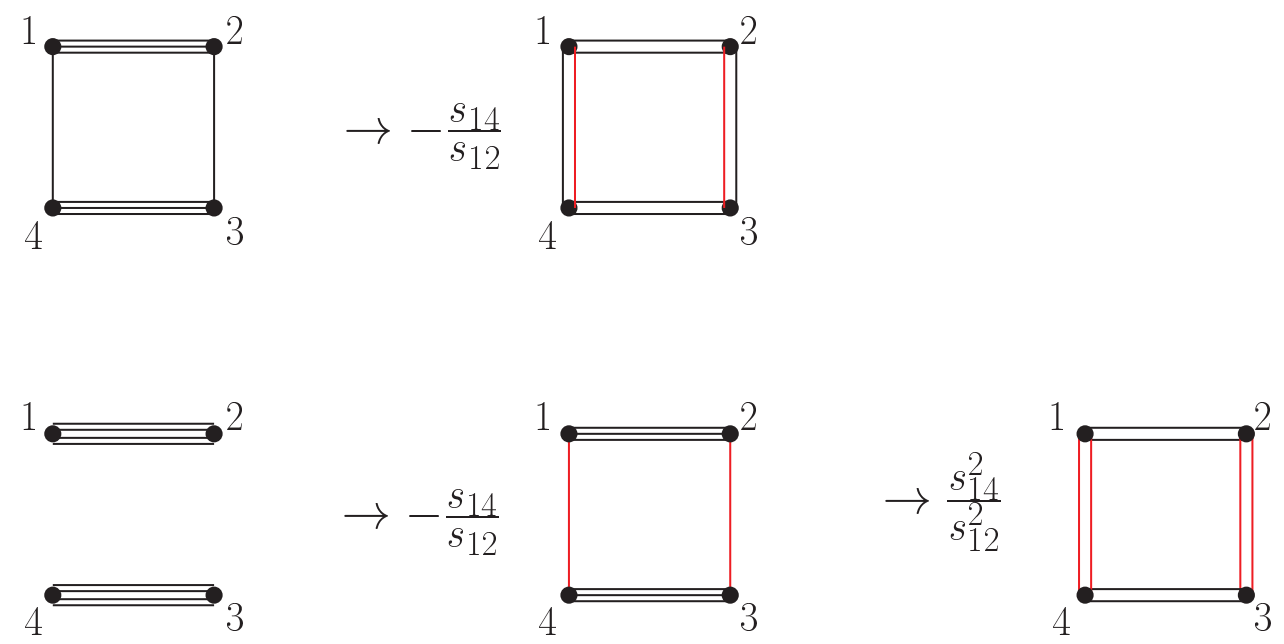

Figure 2. The diagrammatic presentation of how CHY-integrands with different pole structures can be related by cross-ratio identities.

while multiplying it two times with $\mathcal{I}_{4}^{\mathrm{T}}$ leads to

$$
\mathcal{I}_{4}^{\mathrm{T}}=\left(\frac{1}{z_{12}^{4} z_{34}^{4}}\right) \times\left(-\frac{s_{14}}{s_{12}} \frac{z_{12} z_{43}}{z_{14} z_{23}}\right)^{2}=\frac{s_{14}^{2}}{s_{12}^{2}} \mathcal{I}_{4}^{\mathrm{S}},
$$

which are diagrammatically shown in figure 2. Hence for four-point case, an evaluation of CHY-integrands with simple poles is sufficient to produce results of all other CHYintegrands with higher-order poles.

The idea encoded in above decomposition procedure is readily generalized to any CHYintegrands of higher-order poles. For a given $n$-point $\mathrm{CHY}$-integrand, the poles as well as the order of poles can be determined by Pole Condition. Whenever there is any higherorder pole $\frac{1}{s_{A}^{\alpha}}$, we can reduce the order of pole by multiplying identities containing that pole $s_{A}$. The decomposition proceeds by iteratively reducing the higher-order poles, until every resulting CHY-integrand is composed of simple poles. In practical computation, this strategy can be carried out only when there are enough identities of any possible poles, and as mentioned, the Pfaffian identity is obviously not a good candidate. However, the cross-ratio identity that appeared in the four-point examples can be generalized to satisfy the computational demand, which we will describe below.

The cross-ratio identities. For a $n$-point scattering system, let us pick up an arbitrary scattering equation $\mathcal{E}_{a}$. For $p, q \neq a$, we can modify the scattering equation as

$$
\begin{aligned}
0=\frac{z_{a q}}{s_{a q}} \mathcal{E}_{a}=1+\sum_{b \neq a, q} \frac{z_{a q}}{s_{a q}} \frac{s_{a b}}{z_{a b}} & =1+\sum_{b \neq a, q, p} \frac{z_{a q}}{s_{a q}} \frac{s_{a b}}{z_{a b}}+\frac{-\sum_{t \neq a, p} s_{a t}}{z_{a p}} \frac{z_{a q}}{s_{a q}} \\
& =\left(1-\frac{z_{a q}}{z_{a p}}\right)+\sum_{b \neq a, q, p} \frac{s_{a b}}{s_{a q}}\left(\frac{z_{a q}}{z_{a b}}-\frac{z_{a q}}{z_{a p}}\right)
\end{aligned}
$$


where in the last step of line one we have rewritten $s_{a p}$ by momentum conservation. Using $z_{i j}-z_{i k}=z_{k j}$, we get

$$
0=\frac{z_{q p}}{z_{a p}}+\sum_{b \neq a, q, p} \frac{s_{a b}}{s_{a q}} \frac{z_{a q} z_{b p}}{z_{a b} z_{a p}} .
$$

Hence the cross-ratio identity for $s_{i j}$-type pole can be formulated as

$$
1=-\sum_{b \neq a, q, p} \frac{s_{a b}}{s_{a q}} \frac{z_{a q} z_{b p}}{z_{a b} z_{q p}} .
$$

Again we remark that in each term of the identity, $z_{i j}$ 's are arranged as cross-ratios, which are invariant under Möbius transformation. The identities (2.10) will be called fundamental cross-ratio identities since all other identities of given pole $s_{i_{1} i_{2} \cdots i_{m}}$ can be derived from them.

Before presenting the cross-ratio identity for a generic pole $s_{i_{1} i_{2} \cdots i_{m}}$, let us show the construction by taking the pole $s_{i j k l}$ as a warm up. The fundamental cross-ratio identity (2.10) can be rewritten as

$$
-s_{i j}=\sum_{b \neq i, j, p} s_{i b} \frac{z_{b p}}{z_{i b}} \frac{z_{i j}}{z_{j p}} .
$$

Let us take an arbitrary $p \neq i, j, k, l$, and consider the following three fundamental crossratio identities

$$
\begin{aligned}
& -s_{i j}=s_{i k} \frac{z_{k p} z_{i j}}{z_{i k} z_{j p}}+s_{i l} \frac{z_{l p} z_{i j}}{z_{i l} z_{j p}}+\sum_{b \neq i, j, k, l, p} s_{i b} \frac{z_{b p} z_{i j}}{z_{i b} z_{j p}}, \\
& -s_{k j}=s_{k i} \frac{z_{i p} z_{k j}}{z_{k i} z_{j p}}+s_{k l} \frac{z_{l p} z_{k j}}{z_{k l} z_{j p}}+\sum_{b \neq i, j, k, l, p} s_{k b} \frac{z_{b p} z_{k j}}{z_{k b} z_{j p}} \\
& -s_{l j}=s_{l i} \frac{z_{i p} z_{l j}}{z_{l i} z_{j p}}+s_{l k} \frac{z_{k p} z_{l j}}{z_{l k} z_{j p}}+\sum_{b \neq i, j, k, l, p} s_{l b} \frac{z_{b p} z_{l j}}{z_{l b} z_{j p}} .
\end{aligned}
$$

It is easy to see that

$$
s_{i k} \frac{z_{k p} z_{i j}}{z_{i k} z_{j p}}+s_{k i} \frac{z_{i p} z_{k j}}{z_{k i} z_{j p}}=s_{i k}, \quad s_{i l} \frac{z_{l p} z_{i j}}{z_{i l} z_{j p}}+s_{l i} \frac{z_{i p} z_{l j}}{z_{l i} z_{j p}}=s_{i l}, \quad s_{k l} \frac{z_{l p} z_{k j}}{z_{k l} z_{j p}}+s_{l k} \frac{z_{k p} z_{l j}}{z_{l k} z_{j p}}=s_{l k},
$$

so summing over the three identities, we obtain

$$
-s_{i j k l}=\sum_{b \neq i, j, k, l, p}\left(s_{i b} \frac{z_{b p} z_{i j}}{z_{i b} z_{j p}}+s_{k b} \frac{z_{b p} z_{k j}}{z_{k b} z_{j p}}+s_{l b} \frac{z_{b p} z_{l j}}{z_{l b} z_{j p}}\right) .
$$

This immediately gives the cross-ratio identity of $s_{i j k l}$-type pole as

$$
1=-\frac{1}{s_{i j k l}} \sum_{b \neq i, j, k, l, p}\left(s_{i b} \frac{z_{b p} z_{i j}}{z_{i b} z_{j p}}+s_{k b} \frac{z_{b p} z_{k j}}{z_{k b} z_{j p}}+s_{l b} \frac{z_{b p} z_{l j}}{z_{l b} z_{j p}}\right) .
$$

Some remarks are in order for identity (2.17). The first is about the factors $z_{i j}, z_{k j}, z_{l j}$ in the numerator of each term respectively. They are crucial for reducing the number of lines that connecting nodes $\{i, j, k, l\}$, hence consequently reducing the degree of pole $s_{i j k l}$ 
by one. This is the key point of our algorithm. The second is about the $p$ index. It could be any one in $\{1,2, \ldots, n\}$ except $i, j, k, l$. Otherwise for instance $p=k$, there would always be a factor $z_{j p} \equiv z_{j k}$ in the denominator of (2.17), such that the number of lines connecting nodes $\{i, j, k, l\}$ will increase by one. So the order of poles will not be reduced. The third is about the $j$ index. In the derivation, we have chosen to fix the index $j$ and consider three fundamental cross-ratio identities for poles $s_{i j}, s_{k j}, s_{l j}$. Such choice breaks the symmetry among indices $\{i, j, k, l\}$, leaving only $S_{3}$ permutation symmetry on $\{i, k, l\}$ manifest. Similarly, the choice of $p$ also breaks the symmetry among remaining indices. As a consequence, different choice of $j, p$ leads to different cross-ratio identity for the same pole $s_{i j k l}$, and during computation we can choose an appropriate one to simplify the decomposition.

The construction of cross-ratio identity for generic pole $\frac{1}{s_{A}}$ follows exactly the same derivation. Let $A$ be a subset of $\{1,2, \ldots, n\}$, and assume $\bar{A}$ to be its complement. Because of momentum conservation, $s_{A}=s_{\bar{A}}$. Then the cross-ratio identity for pole $s_{A}$ with selected index $j \in A$ and $p \in \bar{A}$ is

$$
1=-\sum_{i \in A \backslash\{j\}} \sum_{b \in \bar{A} \backslash\{p\}} \frac{s_{i b}}{s_{A}} \frac{z_{b p} z_{i j}}{z_{i b} z_{j p}} \equiv \mathbb{I}_{n}[A, j, p],
$$

where $A \backslash\{j\}$ denotes the set $A$ extracting the element $j$. In the notation $\mathbb{I}_{n}[A, j, p], A$ is the subset associated to the pole $s_{A}$ of identity, and $j, p$ are explicitly written down to emphasize the special choice. We remark again that, the cross-ratio identity is invariant under permutation on $A \backslash\{j\}$ as well as permutation on $\bar{A} \backslash\{p\}$, so there are in total $k(n-k)$ different identities for the pole $s_{A}$ if $A$ is a length- $k$ subset.

With the general construction of cross-ratio identities, we can now implement an algorithm for the decomposition of any CHY-integrands with higher-order poles. In the next section, we will present a systematic decomposition algorithm, but now let us follow two examples to explore some details during the decomposition.

Two examples. The first example considers the CHY-integrand

$$
\mathcal{I}_{6}^{[1]}=\frac{1}{z_{12}^{3} z_{23} z_{34}^{3} z_{45} z_{56}^{3} z_{61}}
$$

with its 4-regular graph shown in figure 3. From the counting of lines among subsets of nodes, we know the result for this CHY-integrand should be

$$
\frac{N\left(s_{i j}\right)}{s_{12}^{2} s_{34}^{2} s_{56}^{2} s_{123} s_{234} s_{345}},
$$

where $N\left(s_{i j}\right)$ is some polynomial function of $s_{i j}$ which is irrelevant for the purpose of the current example. So in order to perform the decomposition, we need to multiply it with a cross-ratio identity of the pole $\frac{1}{s_{12}}$, an identity of the pole $\frac{1}{s_{34}}$ and an identity of the pole $\frac{1}{s_{5}}$. Since for each $s_{i j}$, there are $2(6-2)=8$ cross-ratio identities, so naively we have $8^{3}=512$ possibilities of multiplying three identities. However, not all of them can successfully decompose $\mathcal{I}_{6}^{[1]}$ into terms with only simple poles, since with the multiplication of $z_{i j}$, new higher-order poles would appear in some terms (as long as there are more than 


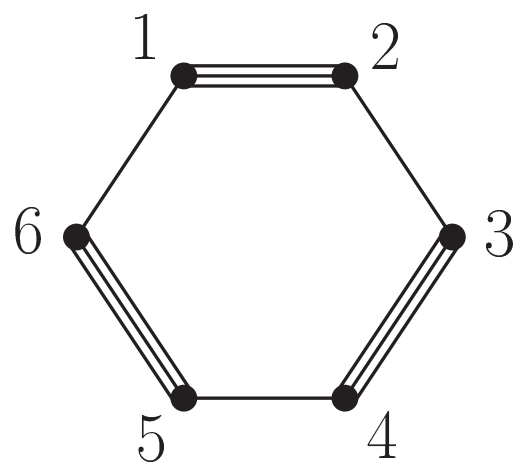

Figure 3. The 4-regular graph of a six-point CHY-integrand with three double poles.

two terms for a new higher-order pole, such that after summing over these terms the new higher-order pole is still canceled.). Of course, the best expectation is that we can find at least one multiplication such that the original CHY-integrand can be decomposed into terms with only simple poles. Fortunately in this example, if taking the following three fundamental cross-ratio identities

$$
\begin{aligned}
& \mathbb{I}_{6}[\{1,2\}, 2,5]=-\left(\frac{s_{13} z_{12} z_{35}}{s_{12} z_{13} z_{25}}+\frac{s_{14} z_{12} z_{45}}{s_{12} z_{14} z_{25}}+\frac{s_{16} z_{12} z_{65}}{s_{12} z_{16} z_{25}}\right), \\
& \mathbb{I}_{6}[\{3,4\}, 3,5]=-\left(\frac{s_{46} z_{65} z_{43}}{s_{34} z_{35} z_{46}}+\frac{s_{14} z_{15} z_{43}}{s_{34} z_{35} z_{41}}+\frac{s_{24} z_{25} z_{43}}{s_{34} z_{35} z_{42}}\right), \\
& \mathbb{I}_{6}[\{5,6\}, 5,1]=-\left(\frac{s_{26} z_{21} z_{65}}{s_{56} z_{51} z_{62}}+\frac{s_{36} z_{31} z_{65}}{s_{56} z_{51} z_{63}}+\frac{s_{46} z_{41} z_{65}}{s_{56} z_{51} z_{64}}\right),
\end{aligned}
$$

i.e.,

$$
1=\mathbb{I}_{6}[\{1,2\}, 2,5] \mathbb{I}_{6}[\{3,4\}, 3,5] \mathbb{I}_{6}[\{5,6\}, 5,1],
$$

then all the resulting $3^{3}=27$ terms after expanding $\mathcal{I}_{6}^{[1]} \times \mathbb{I}_{6}[\{1,2\}, 2,5] \times \mathbb{I}_{6}[\{3,4\}, 3,5] \times$ $\mathbb{I}_{6}[\{5,6\}, 5,1]$ are CHY-integrands with only simple poles. By using integration rules for simple poles, it is confirmed that summing over these 27 terms indeed produces correct answer.

The second example considers the CHY-integrand

$$
\mathcal{I}_{6}^{[2]}=\frac{1}{z_{12}^{4} z_{34}^{3} z_{45} z_{56}^{3} z_{63}}
$$

with its 4-regular graph shown in figure 4 . Again the result can be inferred as

$$
\frac{N\left(s_{i j}\right)}{s_{12}^{3} s_{34}^{2} s_{56}^{2} s_{123} s_{124} s_{125} s_{126}},
$$

where $N\left(s_{i j}\right)$ is another polynomial function of $s_{i j}$. So we need to multiply it with four fundamental cross-ratio identities, two of the pole $\frac{1}{s_{12}}$ (counting multiplicity), one of the pole $\frac{1}{s_{34}}$ and one of the pole $\frac{1}{s_{56}}$. Since each identity contains 3 terms, after multiplying four identities the original CHY-integrand would be decomposed into $3^{4}=81$ terms.

For each $s_{i j}$, there are 8 cross-ratio identities. We have gone through all $\frac{8(8+1)}{2} \times 8 \times 8=$ 2304 possible multiplications of four fundamental cross-ratio identities which have the poles 


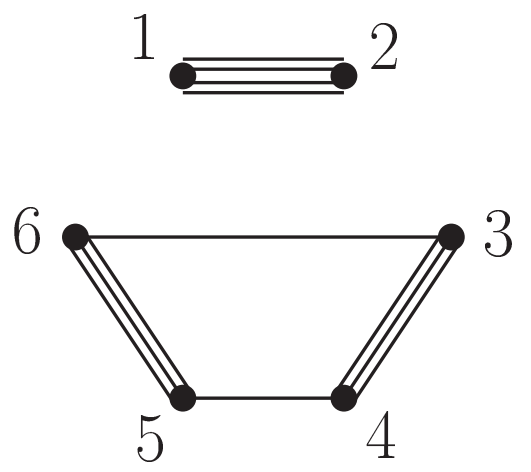

Figure 4. The 4-regular graph of a six-point CHY-integrand with one triple pole and two double poles.

$\frac{1}{s_{12}^{2} s_{34} s_{56}}$, and find none of them can decompose CHY-integrand (2.23) into 81 terms with only simple poles. The best situation is that, 4 out of 81 terms are CHY-integrands with a double pole while the remaining 77 terms with simple poles. Such a multiplication can be constructed from the following four cross-ratio identities

$$
\begin{aligned}
& \mathbb{I}_{6}[\{1,2\}, 2,5]=-\left(\frac{s_{13} z_{12} z_{35}}{s_{12} z_{13} z_{25}}+\frac{s_{14} z_{12} z_{45}}{s_{12} z_{14} z_{25}}+\frac{s_{16} z_{12} z_{65}}{s_{12} z_{16} z_{25}}\right), \\
& \mathbb{I}_{6}[\{1,2\}, 1,5]=-\left(\frac{s_{23} z_{21} z_{35}}{s_{12} z_{15} z_{23}}+\frac{s_{24} z_{21} z_{45}}{s_{12} z_{15} z_{24}}+\frac{s_{26} z_{21} z_{65}}{s_{12} z_{15} z_{26}}\right), \\
& \mathbb{I}_{6}[\{3,4\}, 4,1]=-\left(\frac{s_{35} z_{51} z_{34}}{s_{34} z_{35} z_{41}}+\frac{s_{36} z_{61} z_{34}}{s_{34} z_{36} z_{41}}+\frac{s_{23} z_{21} z_{34}}{s_{34} z_{32} z_{41}}\right), \\
& \mathbb{I}_{6}[\{5,6\}, 6,1]=-\left(\frac{s_{25} z_{21} z_{56}}{s_{56} z_{52} z_{61}}+\frac{s_{35} z_{31} z_{56}}{s_{56} z_{53} z_{61}}+\frac{s_{45} z_{41} z_{56}}{s_{56} z_{54} z_{61}}\right) .
\end{aligned}
$$

After multiplying $\mathbb{I}_{6}[\{1,2\}, 2,5] \times \mathbb{I}_{6}[\{1,2\}, 1,5] \times \mathbb{I}_{6}[\{3,4\}, 4,1] \times \mathbb{I}_{6}[\{5,6\}, 6,1]$ to $\mathcal{I}_{6}^{[2]}$, we get 81 terms, while the following four terms contain double pole, explicitly as

$$
-\frac{s_{14} s_{24} s_{35} s_{36}}{s_{12}^{2} s_{34} s_{56}} \frac{z_{13} z_{45}}{z_{12}^{2} z_{14}^{2} z_{15} z_{24} z_{25} z_{34}^{2} z_{35} z_{36}^{2} z_{56}^{2}}, \quad-\frac{s_{14} s_{24} s_{35}^{2}}{s_{12}^{2} s_{34} s_{56}} \frac{z_{13} z_{45}}{z_{12}^{2} z_{14}^{2} z_{16} z_{24} z_{25} z_{34}^{2} z_{35}^{2} z_{36} z_{56}^{2}}
$$

with double pole $s_{124}$, and

$$
-\frac{s_{16} s_{26} s_{35}^{2}}{s_{12}^{2} s_{34} s_{56}} \frac{z_{13}}{z_{12}^{2} z_{14} z_{16}^{2} z_{25} z_{26} z_{34}^{2} z_{35}^{2} z_{36} z_{45}}, \quad-\frac{s_{16} s_{26} s_{35} s_{45}}{s_{12}^{2} s_{34} s_{56}} \frac{1}{z_{12}^{2} z_{16}^{2} z_{25} z_{26} z_{34}^{2} z_{35} z_{36} z_{45}^{2}}
$$

with double pole $s_{126}$. Note that $s_{124}$ and $s_{126}$ are not double poles of original CHYintegrand, so it should not present in the final answer. The two terms of each double pole guarantee the cancelation. For a complete decomposition, we can further multiply the cross-ratio identity

$$
\mathbb{I}_{6}[\{1,2,4\}, 2,3]=-\left(\frac{s_{15} z_{12} z_{53}}{s_{124} z_{15} z_{23}}+\frac{s_{45} z_{42} z_{53}}{s_{124} z_{23} z_{45}}+\frac{s_{16} z_{12} z_{63}}{s_{124} z_{16} z_{23}}+\frac{s_{46} z_{42} z_{63}}{s_{124} z_{23} z_{46}}\right),
$$

to the two terms in (2.26), and the identity

$$
\mathbb{I}_{6}[\{1,2,6\}, 2,3]=-\left(\frac{s_{14} z_{12} z_{43}}{s_{126} z_{14} z_{23}}+\frac{s_{46} z_{62} z_{43}}{s_{126} z_{23} z_{64}}+\frac{s_{15} z_{12} z_{53}}{s_{126} z_{15} z_{23}}+\frac{s_{56} z_{53} z_{62}}{s_{126} z_{23} z_{65}}\right)
$$


to the two terms in (2.27). Then the four terms with double poles can be further decomposed into terms with simple poles. Hence the CHY-integrand (2.23) is decomposed into $77+4 \times 4=93 \mathrm{CHY}$-integrands of simple poles, and summing over these 93 terms indeed produces the correct answer.

The second example clearly shows that, for generic CHY-integrand of complicated higher-order poles, it is usually not possible to completely decompose it within one step. Even after trying all possible multiplication of cross-ratio identities, there would be some resulting CHY-integrands which need a second and even more steps on cross-ratio identity decomposition. Another thing is about the various cross-ratio identities for the same pole $s_{A}$. To compensate the higher-order pole $s_{A}$, different cross-ratio identities provide different decomposition. Some cross-ratio identities will reduce the degree of higher-order poles in each resulting terms while some will introduce other higher-order poles. These suggest us to implement a decomposition algorithm step by step, and a complete decomposition could be guaranteed only if there are appropriate cross-ratio identities to reduce, or at least not increase, the degree of higher-order poles in each step. Since there are pretty much cross-ratio identities for a pole $s_{A}$, and also various possibilities of decomposition steps, it seems to give us enough information such as the decomposition procedure can always continue until we get a decomposition with CHY-integrands of simple poles.

With above preparations, we are ready to propose a systematic decomposition algorithm in the following section.

\section{A systematic decomposition algorithm}

\subsection{The algorithm}

In order to decompose a CHY-integrand $\mathcal{I}\left(z_{i j}\right)$ of higher-order poles into terms with only simple poles by cross-ratio identities, we can start from an arbitrary higher-order pole $s_{A}$ and multiply an appropriate cross-ratio identity of pole $s_{A}$ to the original CHY-integrand. This leads to several CHY-integrands with the order of higher-order poles reduced. ${ }^{3}$ For each resulting CHY-integrand, we again reduce the order of poles by multiplying an appropriate cross-ratio identity, and iteratively perform this procedure until all resulting terms contain simple poles. Here we present a systematic decomposition algorithm aims to decompose any CHY-integrand of higher-order poles into terms with only simple poles in finite steps.

Let us start from a generic $n$-point CHY-integrand $\mathcal{I}\left(z_{i j}\right)$, as a rational function of $z_{i j}$, as

$$
\mathcal{I}=\frac{1}{\prod_{1 \leq i<j \leq n} z_{i j}^{\beta_{i j}}},
$$

In the 4-regular graph representation, the $\beta_{i j}$ is represented by lines connecting nodes $z_{i}, z_{j}$. A positive integer $\beta_{i j}$ is represented by the corresponding number of solid lines, a negative integer $\beta_{i j}$ (which stands for a non-trivial numerator) is represented by the corresponding

\footnotetext{
${ }^{3}$ There are subtleties that require a careful treatment and we will discuss them along this section.
} 
number of dashed lines. In order to respect the Möbius invariance, for each node, the number of connected solid lines minus the number of connected dashed lines is four.

For the length- $n$ set $\{1,2, \ldots, n\}$, since a subset is considered to be equivalent to its complement due to momentum conservation, we have the following independent subsets $\widetilde{A}$,

- If $n$ is odd, $\widetilde{A}_{\alpha}=\left\{i_{1}, i_{2}, \ldots, i_{k}\right\}, \quad i_{1}, i_{2}, \ldots, i_{k} \in\{1,2, \ldots, n\}, 2 \leq k \leq\left\lfloor\frac{n}{2}\right\rfloor$,

- If $n$ is even, $\widetilde{A}_{\alpha}=\left\{i_{1}, i_{2}, \ldots, i_{k}\right\}, \quad i_{1}, i_{2}, \ldots, i_{k} \in\{1,2, \ldots, n\}$, for $2 \leq k \leq\left\lfloor\frac{n}{2}\right\rfloor-1$, and $i_{1}=1, i_{2}, \ldots, i_{k} \in\{2, \ldots, n\}$ for $k=\left\lfloor\frac{n}{2}\right\rfloor$.

For a subset $\widetilde{A}_{\alpha}=\left\{i_{1}, i_{2}, \ldots, i_{k}\right\}$, the number of lines $\mathbb{L}\left[\widetilde{A}_{\alpha}\right]$ connecting nodes $z_{i_{1}}, z_{i_{2}}, \ldots, z_{i_{k}}$ is given by

$$
\mathbb{L}\left[\widetilde{A}_{\alpha}\right]=\sum_{i^{\prime}, j^{\prime} \in \widetilde{A}_{\alpha}} \beta_{i^{\prime} j^{\prime}}
$$

so the pole index $\chi_{\widetilde{A}_{\alpha}}$ for this subset is

$$
\chi_{\widetilde{A}_{\alpha}}=\mathbb{L}\left[\widetilde{A}_{\alpha}\right]-2\left(\left|\widetilde{A}_{\alpha}\right|-1\right)=\left(\sum_{i^{\prime}, j^{\prime} \in \widetilde{A}_{\alpha}} \beta_{i^{\prime} j^{\prime}}\right)-2(k-1) .
$$

From the CHY-integrand (3.1), we can directly read out the pole index for every subset. Each subset $\widetilde{A}_{\alpha}$ corresponds to a pole $\frac{1}{s_{\alpha}}$. From the Pole Condition follows that, if $\chi_{\widetilde{A}_{\alpha}}<0$, the pole will not present in the final result, while if $\chi_{\widetilde{A}_{\alpha}}=0$, a simple pole $\frac{1}{s_{\alpha}}$ will present, and if $\chi_{\widetilde{A}_{\alpha}}>0$, a pole of order $\frac{1}{s_{\alpha}^{\chi+1}}$ will appear in the final result.

Assuming that for a given CHY-integrand $\mathcal{I}$, there are $m$ independent subsets $A_{\alpha_{1}}, A_{\alpha_{2}}, \ldots, A_{\alpha_{m}}$ with $\chi_{A_{\alpha_{i}}} \geq 0$, we define the order of poles of the CHY-integrand as

$$
\Upsilon[\mathcal{I}]=\sum_{i=1}^{m} \chi_{A_{\alpha_{i}}}
$$

which can be readily computed from $\beta_{i j}$ by using (3.3). The $\Upsilon$ is the number of poles to be compensated by cross-ratio identities, i.e., in order to completely decompose a CHY-integrand, we need to multiply at least $\Upsilon$ cross-ratio identities. $\Upsilon[\mathcal{I}]=0$ means the corresponding CHY-integrand $\mathcal{I}$ contains only simple poles. We will use it as a criteria in the decomposition algorithm.

Before stating the algorithm, let us have a look at the cross-ratio identities. For a generic pole $s_{i_{1} i_{2} \cdots i_{k}}$, from definition (2.18) we know that there are $k(n-k)$ identities

$$
\begin{aligned}
\mathbb{I}_{n}\left[\left\{i_{1}, i_{2}, \ldots, i_{k}\right\}, j, p\right], & j \in\left\{i_{1}, i_{2}, \ldots, i_{k}\right\}, \\
& p \in\{1,2, \ldots, n\} \backslash\left\{i_{1}, i_{2}, \ldots, i_{k}\right\} .
\end{aligned}
$$

Each identity gives a different decomposition of CHY-integrand with higher-order pole of $s_{i_{1} i_{2} \cdots i_{k}}$, and we need to choose an appropriate one in the algorithm.

Now let us state the decomposition algorithm. For a generic CHY-integrand $\mathcal{I}$, we

1. Compute the order of poles $\Upsilon[\mathcal{I}]$. If $\Upsilon[\mathcal{I}]=0$, return $\mathcal{I}$ itself. If $\Upsilon[\mathcal{I}]>0$, list all independent subsets with $\chi>0$ (assuming there are $m^{\prime}$ )

$$
A_{\alpha_{1}}^{\prime}, \quad A_{\alpha_{2}}^{\prime}, \quad \ldots, \quad A_{\alpha_{m^{\prime}}}^{\prime} .
$$


2. Take the first $A_{\alpha_{1}}^{\prime}$, and list all $\left|A_{\alpha_{1}}^{\prime}\right|\left(n-\left|A_{\alpha_{1}}^{\prime}\right|\right)$ cross-ratio identities of $s_{\alpha_{1}}$,

$$
\mathbb{I}_{n}\left[A_{\alpha_{1}}^{\prime}, j, p\right] \quad \text { where } \quad j \in A_{\alpha_{1}}^{\prime}, \quad p \in\{1,2, \ldots, n\} \backslash A_{\alpha_{1}}^{\prime} .
$$

3. Decompose the CHY-integrand $\mathcal{I}$ with the first cross-ratio identity in step 2 ,

$$
\mathcal{I}=\mathcal{I} \times \mathbb{I}_{n}\left[A_{\alpha_{1}}^{\prime}, j, p\right]=\sum_{\ell} c_{\ell} \mathcal{I}_{\ell}^{\prime}
$$

where $\mathcal{I}^{\prime}$ 's are resulting new CHY-integrands, and $c_{\ell}$ 's are rational functions of Mandelstam variables.

4. Compute all $\Upsilon\left[\mathcal{I}^{\prime}\right]$,

- If all $\Upsilon\left[\mathcal{I}^{\prime}\right]<\Upsilon[\mathcal{I}]$, return $\sum_{\ell} c_{\ell} \mathcal{I}_{\ell}^{\prime}$,

- If any $\Upsilon\left[\mathcal{I}^{\prime}\right] \geq \Upsilon[\mathcal{I}]$, try the second cross-ratio identity in step 2 and so on, until find a cross-ratio identity satisfying all $\Upsilon\left[\mathcal{I}^{\prime}\right]<\Upsilon[\mathcal{I}]$. By this way, the order of poles of CHY-integrand is at least reduced by one.

- If we can always find a cross-ratio identity such that all $\Upsilon\left[\mathcal{I}^{\prime}\right]<\Upsilon[\mathcal{I}]$, then after at most $\Upsilon[\mathcal{I}]$ steps, the CHY-integrand can be decomposed into terms with only simple poles. This happens for some CHY-integrands but not for all. If after running over all cross-ratio identities of the pole $s_{\alpha_{1}}$, we still can not find an identity such that all $\Upsilon\left[\mathcal{I}^{\prime}\right]<\Upsilon[\mathcal{I}]$, then start from the first cross-ratio identity in step 2 again, but now stop at an identity such that all $\Upsilon\left[\mathcal{I}^{\prime}\right] \leq \Upsilon[\mathcal{I}]$. By this way, some of $\mathcal{I}^{\prime}$ would have the same order of poles as $\mathcal{I}$ but different rational functions of $z_{i j}$. Anyway we return $\sum_{\ell} c_{\ell} \mathcal{I}_{\ell}^{\prime}$.

After above procedure, we get $\mathcal{I}=\sum_{\ell} c_{\ell} \mathcal{I}_{\ell}^{\prime}$. Then let each $\mathcal{I}_{\ell}^{\prime}$ go through the procedure recursively, until all resulting $\mathrm{CHY}$-integrands contain only simple poles. If after some steps (larger than $\Upsilon[\mathcal{I}]$ ), the terms of higher-order poles in resulting CHY-integrands keep growing, then we shall restart the algorithm again, and choose $A_{\alpha_{2}}^{\prime}$ to start the decomposition, etc. The whole algorithm will end in finite steps, with the judgement that all $\Upsilon\left[\mathcal{I}^{\prime}\right]=0$. Above algorithm can be easily implemented in Mathematica.

\subsection{An illustrative example}

As a highly non-trivial example to illustrate the above mentioned algorithm, let us consider the CHY-integrand

$$
\mathcal{I}_{8}=\frac{1}{z_{12}^{4} z_{34} z_{45}^{3} z_{56} z_{67}^{3} z_{78} z_{38}^{3}},
$$

with its 4-regular graph shown in figure 5 . It can be computed that

$$
\Upsilon\left[\mathcal{I}_{8}\right]=8,
$$

and all subsets with $\chi>0$ are list below,

$$
\{1,2\}, \quad\{3,8\}, \quad\{4,5\}, \quad\{6,7\}, \quad\{1,2,3,8\}, \quad\{1,2,4,5\}, \quad\{1,2,6,7\},
$$




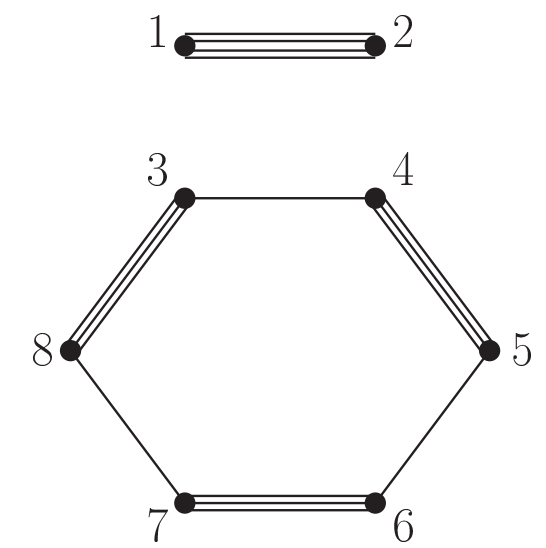

Figure 5. The 4-regular graph of an eight-point CHY-integrand.

where $\chi_{\{1,2\}}=2$ and $\chi=1$ for the remaining six subsets. Let us start from subset $\{3,8\}$ and multiply $\mathcal{I}_{8}$ with a cross-ratio identity of $s_{38} \cdot{ }^{4}$ There are in total $2(8-2)=12$ cross-ratio identities for pole $s_{38}$, list as follows,

$$
\begin{array}{llllll}
\mathbb{I}_{8}[\{3,8\}, 3,1], & \mathbb{I}_{8}[\{3,8\}, 3,2], & \mathbb{I}_{8}[\{3,8\}, 3,4], & \mathbb{I}_{8}[\{3,8\}, 3,5], & \mathbb{I}_{8}[\{3,8\}, 3,6], & \mathbb{I}_{8}[\{3,8\}, 3,7], \\
\mathbb{I}_{8}[\{3,8\}, 8,1], & \mathbb{I}_{8}[\{3,8\}, 8,2], & \mathbb{I}_{8}[\{3,8\}, 8,4], & \mathbb{I}_{8}[\{3,8\}, 8,5], & \mathbb{I}_{8}[\{3,8\}, 8,6], & \mathbb{I}_{8}[\{3,8\}, 8,7] .
\end{array}
$$

Each identity contains five terms, so after decomposition we get five terms

$$
\mathcal{I}_{8}=c_{1} \mathcal{I}_{8}^{\prime[1]}+c_{2} \mathcal{I}_{8}^{[2]}+c_{3} \mathcal{I}_{8}^{\prime[3]}+c_{4} \mathcal{I}_{8}^{\prime[4]}+c_{5} \mathcal{I}_{8}^{\prime[5]} .
$$

Let us take for example the first identity for decomposition, and compute the order of poles of resulting five terms, as

$$
\Upsilon\left[\mathcal{I}_{8}^{\prime[1]}\right]=4, \quad \Upsilon\left[\mathcal{I}_{8}^{\prime[2]}\right]=7, \quad \Upsilon\left[\mathcal{I}_{8}^{\prime[3]}\right]=7, \quad \Upsilon\left[\mathcal{I}_{8}^{\prime[4]}\right]=8, \quad \Upsilon\left[\mathcal{I}_{8}^{\prime[5]}\right]=8 .
$$

It dose not satisfy the condition that all $\Upsilon\left[\mathcal{I}_{8}^{\prime[i]}\right]<8$, so we look for the next identity. It can be found that the first identity satisfying this condition is $\mathbb{I}_{8}[\{3,8\}, 3,6],{ }^{5}$ with which we have

$$
\Upsilon\left[\mathcal{I}_{8}^{\prime[1]}\right]=7, \quad \Upsilon\left[\mathcal{I}_{8}^{\prime[2]}\right]=7, \quad \Upsilon\left[\mathcal{I}_{8}^{\prime[3]}\right]=6, \quad \Upsilon\left[\mathcal{I}_{8}^{[4]}\right]=6, \quad \Upsilon\left[\mathcal{I}_{8}^{\prime[5]}\right]=4 .
$$

This finishes the Round 1 decomposition, and we should go through Round 2 decomposition with each $\mathcal{I}_{8}^{\prime[i]}$ going through the strategy, until all resulting terms satisfying $\Upsilon\left[\mathcal{I}_{8}^{\prime[i]}\right]=0$. Below is a table showing the number \#[ALL] of resulting terms and the number \#[H] of terms of higher-order poles in each Round decomposition,

\begin{tabular}{|c|c|c|c|c|c|c|c|c|}
\hline & Round 1 & Round 2 & Round 3 & Round 4 & Round 5 & Round 6 & Round 7 & Round 8 \\
\hline$\#[\mathrm{ALL}]$ & 5 & 25 & 121 & 613 & 2779 & 7543 & 9914 & 9922 \\
\hline$\#[\mathrm{H}]$ & 5 & 25 & 121 & 464 & 615 & 301 & 2 & 0 \\
\hline
\end{tabular}

\footnotetext{
${ }^{4}$ According to the algorithm, we have taken the first subset $\{1,2\}$ to start the decomposition. However after 10 rounds of decompositions, we still can not get a result with CHY-integrands of only simple poles. Then we restart the algorithm with the second subset $\{3,8\}$.

${ }^{5}$ In fact, among the 12 identities, there are four satisfying the request. But since we only need to find one identity, we do not need to check the remaining ones when a required one is obtained.
} 
It can be seen from the table that, after four rounds of decomposition, some resulting terms are already those of simple poles. After five rounds of decomposition, terms of higher-order poles start decreasing, until to the round 8 decomposition, where all terms of higher-order poles are reduced, leaving $9922 \mathrm{CHY}$-integrands of simple poles. Computing these 9922 terms via integration rules of simple poles takes a few minutes by MATHEMATICA in a laptop, and the result is confirmed numerically.

This algorithm can be applied to higher- $n$ scattering process without difficulty. The efficiency of decomposition mostly depends on the number of terms of higher-order poles in each round decomposition but not the number $n$ of scattering points. If $\Upsilon$ of original CHYintegrand is not very large, the algorithm can be easily finished in a short time. However, if $\Upsilon$ is large (for instance $\Upsilon>10$ ), the decomposition can still proceed, but might take some time.

\section{The $\Lambda$-algorithm and the cross-ratio identities}

In the previous section, we apply the cross-ratio identities to the systematic decomposition of CHY-integrand with higher-order poles. For CHY-integrands with large $n$ and $\Upsilon$, the resulting terms of simple poles can easily reach a number of millions, hence slow the computation. For those CHY-integrands where decomposition algorithm is significantly slow, we can nevertheless combine the cross-ratio identities with $\Lambda$-algorithm, to pursue a more efficient realization. In this section, we will describe such a combination.

The $\Lambda$-algorithm has been recently developed by one of the authors to compute CHYintegrands. It has some interesting features as it can support up to three off-shell particles as well as it factorizes the original graph representing the CHY-integrand into sub-graphs with less number of vertices by means of an iterative algorithm. Nevertheless, it depends on the gauge-fixing and it does not work on singular configurations. At some point on the iterative process one usually reach some sub-graphs containing those singular configurations and then the algorithm cease to work. Here we show how the $\Lambda$-algorithm is improved by using the cross-ratio identities on graphs containing singular configurations. In addition, we find some recurrence relations for particular types of CHY-integrands. Ultimately, the cross-ratio identities in conjunction with the $\Lambda$-algorithm provide a more efficient and systematic way to deal with amplitudes with a large number of particles.

\subsection{Some notations}

For reader's convenience, let us briefly introduce some notations here, which will be useful in the computation of some non-trivial examples soon after. We define the stripped Mandelstam variables $\widetilde{s}_{a_{1} \ldots a_{m}}$ as

$$
\widetilde{s}_{a_{1} \ldots a_{m}}:=\sum_{a_{i}<a_{j}}^{m} k_{a_{i}} \cdot k_{a_{j}},
$$

which equals to the standard Mandelstam variables $s_{a_{1} \ldots a_{m}}=\left(k_{a_{1}}+\cdots+k_{a_{m}}\right)^{2} / 2$ when all $k_{a_{i}}$ 's are massless. We also follow the conventional definition

$$
k_{a_{1} \ldots a_{m}}:=k_{a_{1}}+k_{a_{2}}+\cdots+k_{a_{m}},
$$


and whenever $\left[a_{1}, \ldots, a_{m}\right]$ is present in $\mathcal{I}(z)$ or $\widetilde{s}$, it stands for the punctured point merged from points $\left\{z_{a_{1}}, \ldots, z_{a_{m}}\right\}$ in graph associated to the massive momentum $k_{a_{1} \ldots a_{m}}$. The colored notation in [21] which is needed to apply the $\Lambda$-algorithm is adopted,

Free Vertex
Anti-line
Massive and fixed Vertex (Puncture)

and for details please refer to [21].

All the results obtained from the $\Lambda$-algorithm can be written as a linear combination of the following fundamental diagrams and its powers

$$
B(a, b \mid c, d):=\overbrace{d}^{c}=\frac{1}{\widetilde{s}_{a c}}+\frac{1}{\widetilde{s}_{a d}},
$$

where $\left\{k_{b}, k_{c}, k_{d}\right\}$ can be off-shell particles. Clearly, $B(a, b \mid c, d)=B(a, b \mid d, c)$.

It is simple to check, using the $\mathcal{E}_{a}$ scattering equation, that

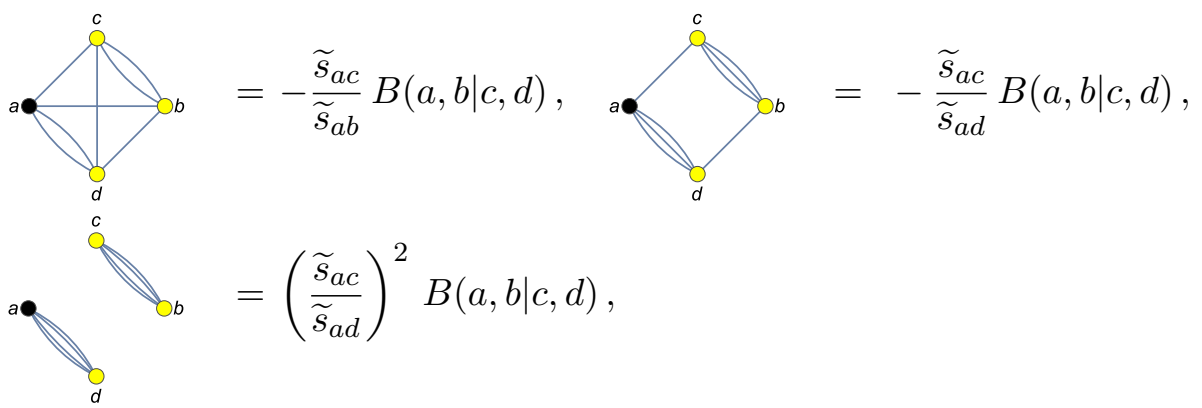

where $\left\{k_{b}, k_{c}, k_{d}\right\}$ could be off-shell particles.

\subsection{A simple example}

Before a general discussion, let us start from a simple but non-trivial six-point example with the following geometry (Parke - Taylor $)^{2} \oplus(\text { Parke }- \text { Taylor })^{2}$ as

$$
\mathcal{I}_{6}(1,2,3 \mid 4,5,6)=\overbrace{5}
$$


where we have set the gauge-fixing so as to avoid singular configurations [21]. All non-zero allowable configurations for this diagram are given by [21]

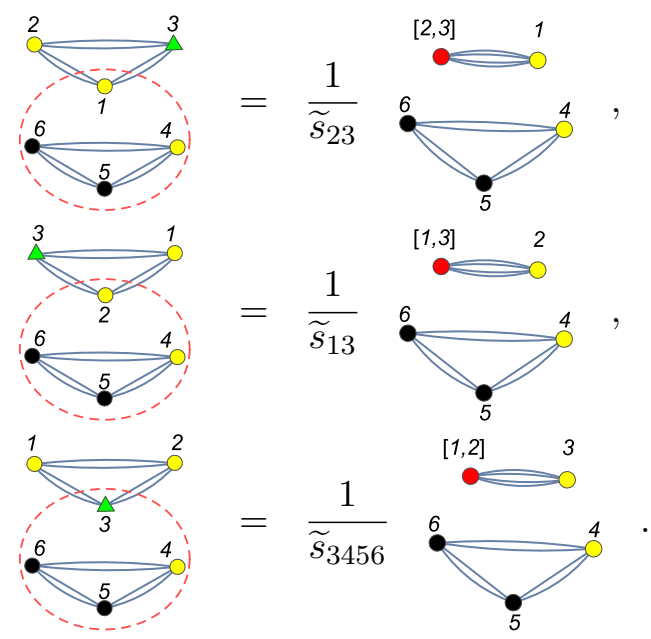

The $\Lambda$-algorithm stops at this step, since we have reached sub-diagrams containing singular configurations

$$
\mathcal{I}_{5}(a, b \mid c, d, e)=\overbrace{a}^{c} c
$$

which needs to be rewritten in terms of non-singular sub-diagrams by using the cross-ratio identities, as we shall explain soon after. Let us remember that in this graph, $\left\{k_{a}, k_{b}, k_{c}\right\}$ can be off-shell.

First of all, notice that only the $\mathcal{E}_{d}$ and $\mathcal{E}_{e}$ scattering equations can be used, since the remaining points are already fixed. Moreover, clearly this graph has a triple pole, $1 / \widetilde{s}_{c d e} 3$. So, it is simple to show that using the $\mathcal{E}_{d}$ and $\mathcal{E}_{e}$ scattering equations one obtains the following cross-ratio identity

$$
\widetilde{s}_{c d e}=\widetilde{s}_{a d}\left(\frac{z_{a b} z_{c d}}{z_{b c} z_{a d}}\right)+\widetilde{s}_{a e}\left(\frac{z_{a b} z_{c e}}{z_{b c} z_{a e}}\right)
$$

which agrees with (2.18). We can represent the identity above by the graph

$$
\widetilde{s}_{c d e}=\widetilde{s}_{a d} \in \int_{a}^{a} \int_{0}^{b}+\widetilde{s}_{a e}
$$

In order to eliminate the triple pole we take the square of (4.8)

$$
\tilde{s}_{c d e}^{2}=\widetilde{s}_{a d}^{2} e \bullet \int_{a====0}^{a} \int_{c}^{b}+\widetilde{s}_{a e}^{2}
$$


Therefore, using this identity over the CHY diagram in (4.6), we obtain the expansion
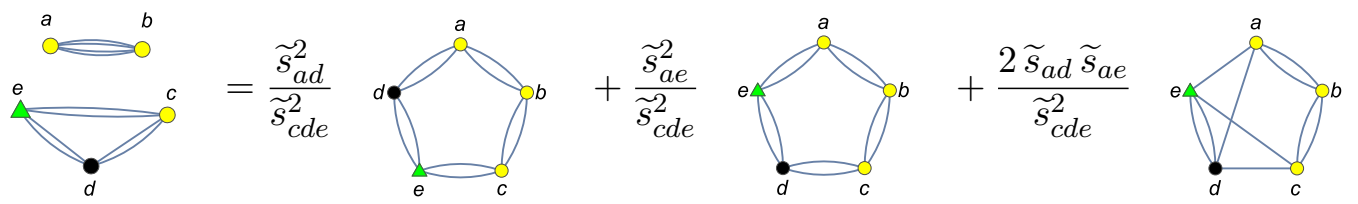

These three graphs are computed straightforwardly from the $\Lambda$-algorithm resulting in,

$$
\begin{aligned}
\mathcal{I}_{5}(a, b \mid c, d, e)= & \frac{\widetilde{s}_{a d}^{2}}{\widetilde{s}_{c d e}^{2}}\left(\frac{B(d, c \mid[a, b], e)}{\widetilde{s}_{c d e}}+\frac{B(d,[b, c] \mid a, e)}{\widetilde{s}_{d e a}}+\frac{B(d, b \mid a,[c, e])}{\widetilde{s}_{c e}}\right) \\
& +\frac{\widetilde{s}_{a e}^{2}}{\widetilde{s}_{c d e}^{2}}\left(\frac{B(d,[a, b] \mid c,, e)}{\widetilde{s}_{c d e}}+\frac{B(d, a \mid[b, c], e)}{\widetilde{s}_{d e a}}+\frac{B(d, b \mid c,[a, e])}{\widetilde{s}_{a e}}\right) \\
& +\frac{2 \widetilde{s}_{a e} \widetilde{s}_{a d}}{\widetilde{s}_{c d e}^{2}}\left(-\frac{\widetilde{s}_{d c}}{\widetilde{s}_{c d e} \widetilde{s}_{d[a, b]}} B(d,[a, b] \mid c, e)-\frac{\widetilde{s}_{d[b, c]}}{\widetilde{s}_{d e a} \widetilde{s}_{d a}} B(d, a \mid[b, c], e)\right) .
\end{aligned}
$$

Hence, the final answer for the CHY-integrand in (4.5) is given in terms of $\mathcal{I}_{5}(a, b \mid c, d, e)$ by the simple expression

$$
\mathcal{I}_{6}(1,2,3 \mid 4,5,6)=\frac{\mathcal{I}_{5}([2,3], 1 \mid 4,5,6)}{\widetilde{s}_{23}}+\frac{\mathcal{I}_{5}([1,3], 2 \mid 4,5,6)}{\widetilde{s}_{13}}+\frac{\mathcal{I}_{5}([1,2], 3 \mid 4,5,6)}{\widetilde{s}_{3456}},
$$

which was checked numerically.

We remark that, for this particular example where we have combined the $\Lambda$-algorithm with the cross-ratio identities, we solved a total of three CHY-integrands. On the other hand, applying directly the cross-ratio identities over the CHY-integrand in (4.5), one must compute ten CHY graphs, So, although the decomposition technique with the cross-ratio identities is a good method by itself, in combination with the $\Lambda$-algorithm it produces an even more efficient approach. The simple example above is enough to show how both methods work out together, and instead of going into harder examples we choose to present a new recurrence relation that can be obtained from this combination.

\section{3 (Parke - Taylor $)^{2} \oplus(\text { Parke }- \text { Taylor })^{2}$ geometry and recurrence relations}

In this subsection, we would like to generalize the discussion in previous subsection to a particular geometry given by two $\mathrm{PT}^{2}$ (i.e., $\mathrm{PT}^{2} \oplus \mathrm{PT}^{2}$ ) $\mathrm{CHY}$-integrands, e.g.,
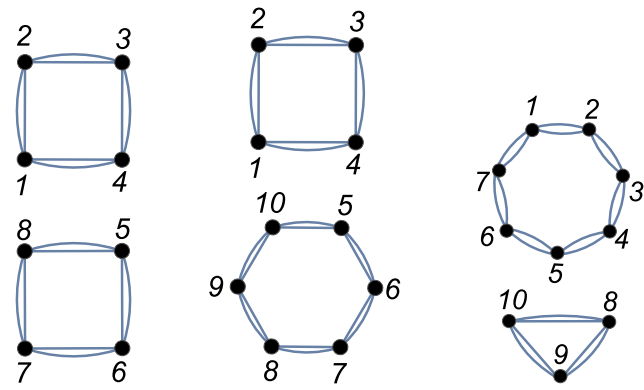

We denote this type of graphs by $\mathcal{I}_{m, n-m}^{\mathrm{PT}^{2} \oplus \mathrm{PT}^{2}}(1, \ldots, m \mid m+1, \ldots, n)$, where $m$ is the number of vertices for the first $\mathrm{PT}^{2}$ sub-graph and $n-m$ is the number of vertices for the second 
one. For instance, the graphs given in (4.12) are denoted as $\mathcal{I}_{4,4}^{\mathrm{PT}^{2} \oplus \mathrm{PT}^{2}}(1,2,3,4 \mid 5,6,7,8)$, $\mathcal{I}_{4,6}^{\mathrm{PT}^{2} \oplus \mathrm{PT}^{2}}(1,2,3,4 \mid 5,6,7,8,9,10) \quad$ and $\quad \mathcal{I}_{7,3}^{\mathrm{PT}^{2} \oplus \mathrm{PT}^{2}}(1,2,3,4,5,6,7 \mid 8,9,10)$, respectively. Note also that for the graphs in (4.5) and (4.6) one has $\mathcal{I}_{6}(1,2,3 \mid 4,5,6)=$ $\mathcal{I}_{3,3}^{\mathrm{PT}^{2} \oplus \mathrm{PT}^{2}}(1,2,3 \mid 4,5,6)$ and $\mathcal{I}_{5}(a, b \mid c, d, e)=\mathcal{I}_{2,3}^{\mathrm{PT}^{2} \oplus \mathrm{PT}^{2}}(a, b \mid c, d, e)$, respectively.

It is very well-known that this type of CHY-integrands is highly non-trivial and one can solve them from the cross-ratio identities. ${ }^{6}$ However, the price to pay is that the number of CHY-integrands of simple poles to be computed is very large. So, in order to partially simplify the decomposition procedure, we seek help from the $\Lambda$-algorithm. Nevertheless, the $\Lambda$-algorithm alone is not enough to obtain the final answer, so we use the cross-ratio identities but now over smaller sub-graphs. Note that for above particular type of diagrams with $\mathrm{PT}^{2} \oplus \mathrm{PT}^{2}$ geometry, after applying the $\Lambda$-algorithm, the resulting sub-graphs are of the same type, i.e. $\mathrm{PT}^{2} \oplus \mathrm{PT}^{2}$, or just $\mathrm{PT}^{2}$. This feature suggests a recurrence relation.

So as to find this recurrence relation, let us consider the following ten-point example
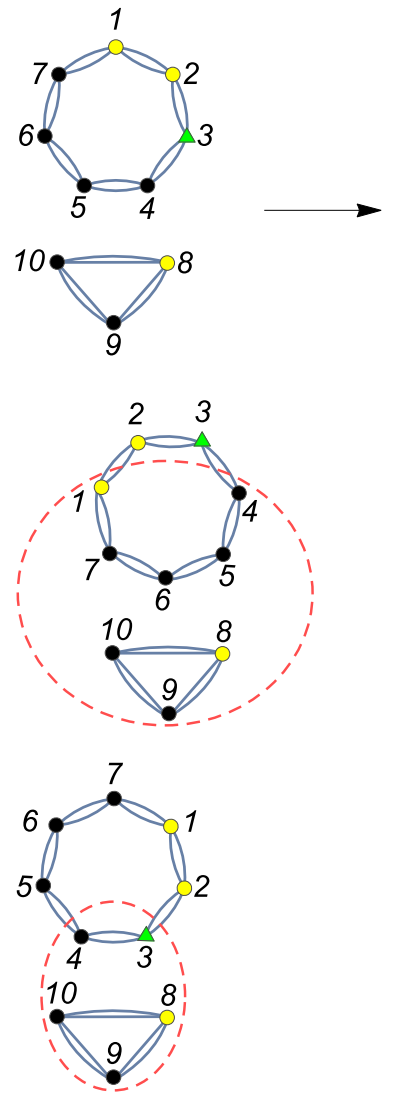

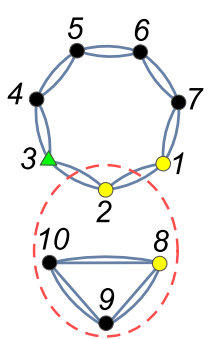

3
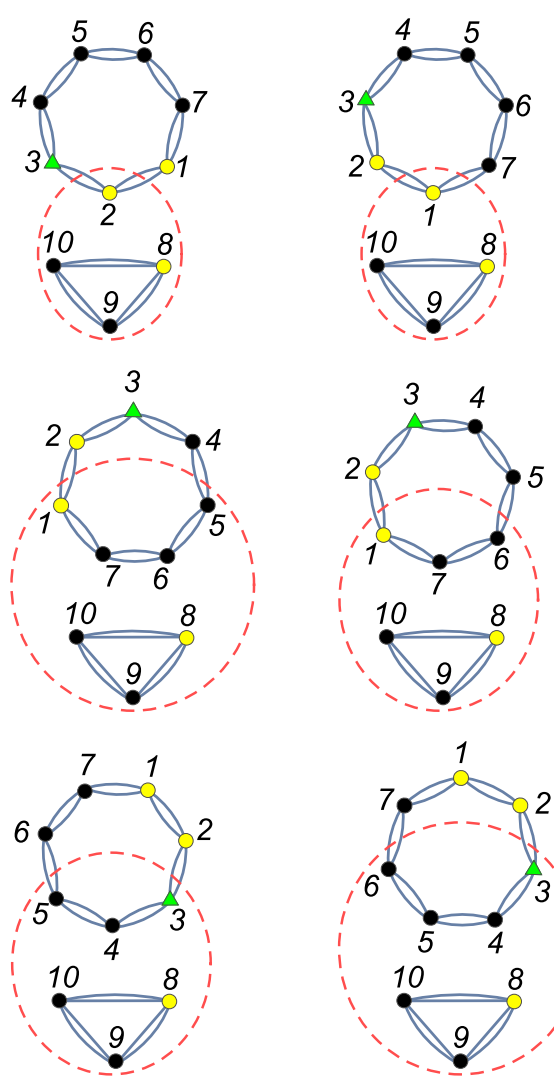
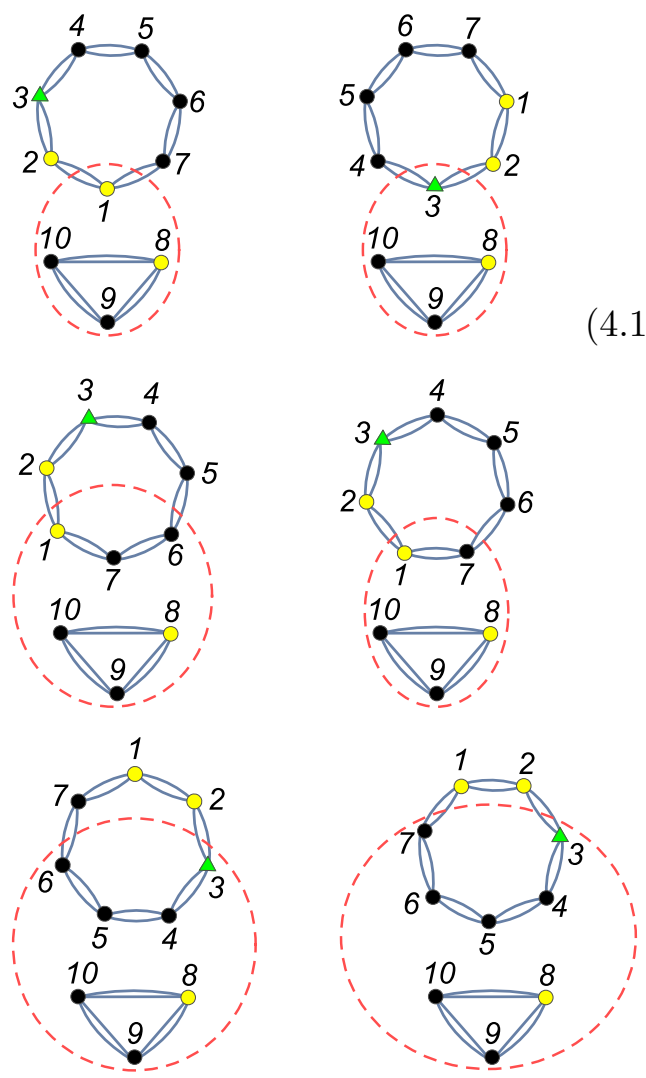

where we have chosen a proper gauge in order to avoid singular configurations and we have drawn all non-zero allowable configurations. Following this example, we can intermediately deduce the new recurrence relation.

\footnotetext{
${ }^{6}$ In fact, if there is only one higher-order poles, either double or triple pole, the decomposition algorithm with cross-ratio identities can produce the result instantly for any points.
} 
By using the notation in appendix $\mathrm{C}$, we make the following definitions. Let $\mathbb{D}_{i}$ be the set of ordered elements given by

$$
\mathbb{D}_{i}:=\{4,5,6, \ldots i+3\}, \quad \text { where } i \in\{1, \ldots, m-3\},
$$

and we define $\mathbb{D}_{0}=\emptyset$. Clearly, $\mathbb{D}_{1}=\{4\}, \mathbb{D}_{2}=\{4,5\}, \mathbb{\Phi}_{3}=\{4,5,6\}$ and so on.

We also denote $\bar{\Phi}_{i}$ as the ordered complement of $\mathbb{\Phi}_{i}$, as

$$
\overline{\mathbb{D}}_{i}:=\{4,5,6, \ldots, m\} \backslash \mathbb{D}_{i},
$$

for example

$$
\bar{\Phi}_{0}=\{4, \ldots, m\}, \quad \bar{\Phi}_{1}=\{5, \ldots, m\}, \quad \ldots, \quad \bar{\Phi}_{m-3}=\emptyset .
$$

With these definitions in mind, the recurrence relation has the form

$$
\begin{aligned}
& \mathcal{I}_{m, n-m}^{\mathrm{PT}^{2} \oplus \mathrm{PT}^{2}}(1,2, \ldots, m \mid m+1, \ldots, n) \\
& =\frac{\mathcal{I}_{2, n-m}^{\mathrm{PT}^{2} \oplus \mathrm{PT}^{2}}([3,4, \ldots, m, 1], 2 \mid m+1, \ldots, n) \times \mathcal{I}_{m}^{\mathrm{PT}^{2}}(1,[2, m+1, \ldots, n], 3 \ldots, m)}{\widetilde{s}_{3,4, \cdots, m, 1}} \\
& \quad+\sum_{i=0}^{m-3} \frac{\mathcal{I}_{m-1-i, n-m}^{\mathrm{PT}^{2} \oplus \mathrm{PT}^{2}}\left(1,\left[2,3, \mathbb{\Phi}_{i}\right], \bar{\Phi}_{i} \mid m+1, \ldots, n\right) \times \mathcal{I}_{3+i}^{\mathrm{PT}^{2}}\left(\left[1, \bar{\Phi}_{i}, m+1, \ldots n\right], 2,3, \mathbb{D}_{i}\right)}{\widetilde{s}_{2,3, \Phi_{i}}} \\
& \quad+\sum_{i=0}^{m-3} \frac{\mathcal{I}_{2+i, n-m}^{\mathrm{PT}^{2} \oplus \mathrm{PT}^{2}}\left(\left[1,2, \bar{\Phi}_{i}\right], 3, \mathbb{\Phi}_{i} \mid m+1, \ldots, n\right) \times \mathcal{I}_{m-i}^{\mathrm{PT}^{2}}\left(1,2,\left[3, \mathbb{\Phi}_{i}, m+1, \ldots n\right], \overline{\mathbb{\Phi}}_{i}\right)}{\widetilde{s}_{3, \Phi_{i}, m+1, \cdots, n}},
\end{aligned}
$$

where remind again that $\left[a_{1}, a_{2}, \ldots, a_{m}\right]$ denotes a massive particle with momentum equaling $\sum_{i=1}^{m} k_{a_{i}}$.

Applying this recurrence relation over the example in (4.13), one obtains (for presentation purpose here we omit the superscript $\mathrm{PT}^{2} \oplus \mathrm{PT}^{2}$ and $\left.\mathrm{PT}^{2}\right)$

$$
\begin{aligned}
\mathcal{I}_{7,3}^{\mathrm{PT}^{2}} \oplus \mathrm{PT}^{2}(1,2,3,4,5,6,7 \mid 8,9,10) \\
=\frac{\mathcal{I}_{2,3}([3,4,5,6,7,1], 2 \mid 8,9,10) \times \mathcal{I}_{7}(1,[2,8,9,10], 3,4,5,6,7)}{\widetilde{s}_{3,4,5,6,7,1}} \\
+\frac{\mathcal{I}_{6,3}(1,[2,3], 4,5,6,7 \mid 8,9,10) \times \mathcal{I}_{3}([1,4,5,6,7,8,9,10], 2,3)}{\widetilde{s}_{2,3}} \\
+\frac{\mathcal{I}_{5,3}(1,[2,3,4], 5,6,7 \mid 8,9,10) \times \mathcal{I}_{4}([1,5,6,7,8,9,10], 2,3,4)}{\widetilde{s}_{2,3,4}} \\
+\frac{\mathcal{I}_{4,3}(1,[2,3,4,5], 6,7 \mid 8,9,10) \times \mathcal{I}_{5}([1,6,7,8,9,10], 2,3,4,5)}{\widetilde{s}_{2,3,4,5}} \\
+\frac{\mathcal{I}_{3,3}(1,[2,3,4,5,6], 7 \mid 8,9,10) \times \mathcal{I}_{6}([1,7,8,9,10], 2,3,4,5,6)}{\widetilde{s}_{2,3,4,5,6}} \\
+\frac{\mathcal{I}_{2,3}(1,[2,3,4,5,6,7] \mid 8,9,10) \times \mathcal{I}_{7}([1,8,9,10], 2,3,4,5,6,7)}{\widetilde{s}_{2,3,4,5,6,7}} \\
+ \\
+\frac{\mathcal{I}_{2,3}([1,2,4,5,6,7], 3 \mid 8,9,10) \times \mathcal{I}_{7}(1,2,[3,8,9,10], 4,5,6,7)}{\widetilde{s}_{3,8,9,10}}
\end{aligned}
$$




$$
\begin{aligned}
& +\frac{\mathcal{I}_{3,3}([1,2,5,6,7], 3,4 \mid 8,9,10) \times \mathcal{I}_{6}(1,2,[3,4,8,9,10], 5,6,7)}{\widetilde{s}_{3,4,8,9,10}} \\
& +\frac{\mathcal{I}_{4,3}([1,2,6,7], 3,4,5 \mid 8,9,10) \times \mathcal{I}_{5}(1,2,[3,4,5,8,9,10], 6,7)}{\widetilde{s}_{3,4,5,8,9,10}} \\
& +\frac{\mathcal{I}_{5,3}([1,2,7], 3,4,5,6 \mid 8,9,10) \times \mathcal{I}_{4}(1,2,[3,4,5,6,8,9,10], 7)}{\widetilde{s}_{3,4,5,6,8,9,10}} \\
& +\frac{\mathcal{I}_{6,3}([1,2], 3,4,5,6,7 \mid 8,9,10) \times \mathcal{I}_{3}(1,2,[3,4,5,6,7,8,9,10])}{\widetilde{s}_{3,4,5,6,7,8,9,10}},
\end{aligned}
$$

which is the right expression for the configurations given in (4.13). The terms $\mathcal{I}_{n}^{\mathrm{PT}^{2}}, n=$ $5,6,7$, can easily be computed from the recurrence relation in (C.7). Now, the terms

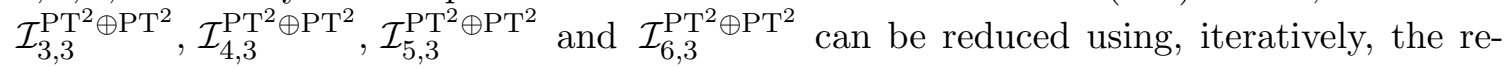
currence relation (4.17). In addition, in appendix D we have given the expressions for these diagrams.

It is important to note that the relation in (4.17) only works for CHY-integrands with $m>2$. In other words, CHY-integrands such as $\mathcal{I}_{2, n-2}^{\mathrm{PT}^{2} \oplus \mathrm{PT}^{2}}(1,2 \mid 3, \ldots, n)$ with $n>4$ under the gauge-fixing as in (4.13) can not be solved just by the $\Lambda$-algorithm, and therefore we should proceed to use the cross-ratio identities. The main idea of the recurrence relation in (4.17) is to use it to straightforwardly reduce the original CHY-integrand $\mathcal{I}_{m, n-m}^{\mathrm{PT}^{2} \oplus \mathrm{PT}^{2}}(1,2, \ldots m, \mid m+1, \ldots, n)$ with $m>2, n-m>2$ to $\mathcal{I}_{2, n^{\prime}-2}^{\mathrm{PT}^{2} \oplus \mathrm{PT}^{2}}$ with $n^{\prime}$ smaller than $n$, and then apply the cross-ratio identities.

For our particular example in (4.13), we were able to reduce the whole expression in (4.18) as a linear combination of $\mathcal{I}_{2,3}^{\mathrm{PT}^{2} \oplus \mathrm{PT}^{2}}(a, b \mid c, d, e)$ diagram (see appendix D). This diagram was solved previously by using the cross-ratio identities, and its answer is given in (4.10).

For the most general case, we must solve the $n=(q+2)$-point CHY-integrand

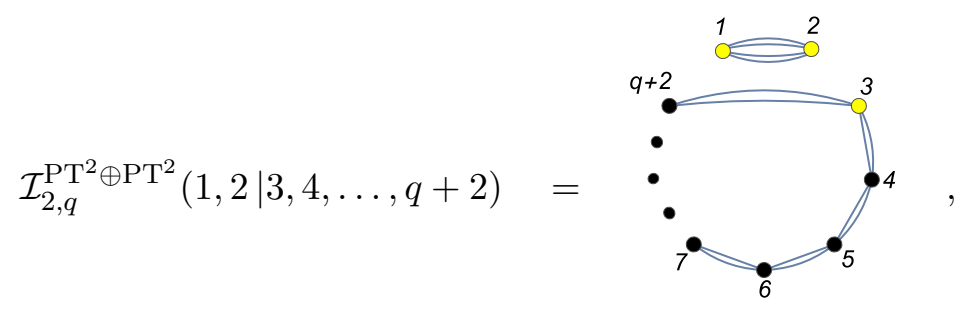

where we use the cross-ratio identities for doing it. Similarly as it was done in section 4.2 for the graph $\mathcal{I}_{2,3}^{\mathrm{PT}^{2} \oplus \mathrm{PT}^{2}}(a, b \mid c, d, e)$, one notes that this graph has a triple pole, $1 / s_{34 \ldots q+2}^{3}$. In order to eliminate this pole, we can use the scattering equations, $\left\{\mathcal{E}_{3}, \mathcal{E}_{4}, \ldots, \mathcal{E}_{q+2}\right\}$, to obtain the cross-ratio identity

$$
\widetilde{s}_{34 \ldots q+2}=\sum_{a=4}^{q+2} \widetilde{s}_{1 a}\left(\frac{z_{12} z_{3 a}}{z_{23} z_{1 a}}\right)
$$


which has $q-1$ elements. Taking the square of this identity one obtains

$$
\begin{aligned}
\widetilde{s}_{34 \ldots q+2}^{2} & =\sum_{a, b=4}^{q+2} \widetilde{s}_{1 a} \widetilde{s}_{1 b}\left(\frac{z_{12} z_{3 a}}{z_{23} z_{1 a}}\right)\left(\frac{z_{12} z_{3 b}}{z_{23} z_{1 b}}\right) \\
& =\sum_{a=4}^{q+2} \widetilde{s}_{1 a}^{2}\left(\frac{z_{12} z_{3 a}}{z_{23} z_{1 a}}\right)+2 \sum_{a<b=4}^{q+2} \widetilde{s}_{1 a} \widetilde{s}_{1 b}\left(\frac{z_{12} z_{3 a}}{z_{23} z_{1 a}}\right)\left(\frac{z_{12} z_{3 b}}{z_{23} z_{1 b}}\right),
\end{aligned}
$$

which has a total number of terms, $q-1+\frac{(q-1)(q-2)}{2}=\frac{q(q-1)}{2}$. Therefore, the CHYintegrand

$$
\mathcal{I}_{2, q}^{\mathrm{PT}^{2} \oplus \mathrm{PT}^{2}}(1,2 \mid 3,4, \ldots, q+2)
$$

is solved in a straightforward manner by computing a number of $\frac{q(q-1)}{2}$ trivial CHYintegrands.

Finally, noticing that the recurrence relation (4.17) is only a consequence of the iterative nature of the $\Lambda$-algorithm, we can build many other relations by using the same method. The only particularity that need to be satisfied in order to have an ordered recurrence is that the graphs under consideration should possess some symmetry such that the cutting process preserves this "symmetry" for the smaller sub-graphs. For instance, for the particular case in (4.13), the original graph is build out from two disjoint pieces, and in an appropriate gauge avoiding singular configurations, all the allowed cuts produce sub-graphs with the same topology as the original graph. Examples where similar recurrence relations can be also obtained are graphs of the type $\left(\mathrm{PT}^{2}\right)^{n}:=\left(\mathrm{PT}^{2}\right) \oplus\left(\mathrm{PT}^{2}\right) \oplus \cdots \oplus\left(\mathrm{PT}^{2}\right)$. Such CHY-integrands could have very large $\Upsilon$, thus it is very necessary to apply $\Lambda$-algorithm before decomposition algorithm with cross-ratio identity. By solely using the $\Lambda$-algorithm we will reach sub-diagrams containing singular configurations where the $\Lambda$-algorithm cease to work and hence stop the recurrence. The remaining sub-diagrams should be then rewritten by means of the cross-ratio identities in order to either solving them directly by integration rules of simple poles or by continuing the cutting process of $\Lambda$-algorithm.

\section{Conclusion}

Based on the extremely simple and efficient method of integration rules for CHY-integrands with only simple poles [23, 24], we propose a systematic decomposition algorithm by use of cross-ratio identities, which can be applied to decompose any CHY-integrand of higherorder poles to those with only simple poles, suitable for evaluating by the integration rules. The basic idea of the algorithm can be described as follows. For any CHY-integrand with higher-order poles, we multiply it with identities which are linear combination of terms as

$$
\frac{s_{A}}{s_{B}} \frac{z_{a_{1} a_{2}} z_{a_{3} a_{4}}}{z_{a_{1} a_{3}} z_{a_{2} a_{4}}}
$$

i.e., cross-ratios of $z_{i j}$. The cross-ratios of $z_{i j}$ reformulate the original CHY-integrands of higher-order poles as new ones with only simple poles which can be evaluated trivially, while the $s_{B}$ in denominator will compensate the extra degrees of higher-order poles, such 
that the final result still possess higher-order poles. This idea is exactly the same as the algorithm proposed in [30], but in [30] identities from monodromy relations are used instead of cross-ratio identities, and we have shown in the appendix B that a decomposition with the former is far less efficient than that with the later for large number of scattering particles and complicated higher-order pole structures, due to the fact that the number of terms in an identity of monodromy relation grows factorially while in cross-ratio identity algebraically.

An eight-point CHY-integrand with one triple pole and six double poles is computed in section 3, resulting in around 10,000 terms of only simple poles, which summed up to produce the amplitude by integration rules, with the evaluation time around a few minutes. The increasing of scattering particles will not affect the computation efficiency much, but the increasing of higher-order poles will significantly increase the number of resulting terms, since in order to compensate an extra $\frac{1}{s_{i_{1} \cdots i_{k}}}$, we need to multiply an identity with $(k-1)(n-k-1)$ terms, and consequently the number of resulting terms will be about $(k-1)(n-k-1)$ times larger. So for CHY-integrands with very large $n$ and complicated higher-order pole structures, the number of resulting CHY-integrands after decomposition will easily reach the size of millions, which slows the computation. ${ }^{7}$ In need of these situations, we seek help from $\Lambda$-algorithm [21, 22]. The $\Lambda$-algorithm rewrites a CHY-integrand as products of lower-point CHY-integrands, until the resulting lower-point ones can not be computed by $\Lambda$-algorithm. We show that for particular type of CHYintegrands, recurrence relations can be deduced to iteratively transform a specific CHYintegrand into lower-point ones which are easy to compute by cross-ratio identities. The combination of $\Lambda$-algorithm with decomposition algorithm makes a more efficient method for evaluating large $n$-particle amplitude by use of cross-ratio identities.

Some aspects are in the following. The CHY-integrand we consider in this paper is quite general, with in fact any possible higher-order poles. Thus the decomposition algorithm is suitable and ready for evaluating Yang-Mills amplitudes and even gravity amplitudes in the context of CHY-formulation, not only in principle but also in practical. Since the crossratio identities deal with CHY-integrands with higher-order poles, while in [29] Feynman rules for higher-order poles are conjectured, it would be interesting to investigate if one can prove those Feynman rules by cross-ratio identities and further derive rules for more higher-order poles. Another interesting problem considers the recurrence relation by $\Lambda$ algorithm. We have in this paper presented a recurrence relation for CHY-integrands with $(\mathrm{PT})^{2} \oplus(\mathrm{PT})^{2}$ geometry, to iteratively rewrite a CHY-integrand to other specific ones which are easy to compute. It would be interesting to generalize the study of recurrence relations to a broader range of CHY-integrands with other complicated geometries where a direct decomposition algorithm would take too much time.

\footnotetext{
${ }^{7}$ Remind that there are many ways of decomposing a CHY-integrand into those with simple poles, and the number of resulting terms depends on the cross-ratio identities multiplied in each step. A cross-ratio identity in one step will affect the number of resulting terms as well as the higher-order pole structure in the next step, while these two factors in together will affect the number of terms in the final step. There is no clear canonical strategy to balance the effects of these two factors and lead to the minimal number of final resulting terms. Thus we have chosen the most straightforward strategy, i.e., multiply the first cross-ratio identity which reduces the order of poles.
} 


\section{Acknowledgments}

We would like to thank Christian Baadsgaard and Song He for helpful conversation. C.C. thanks to the High Energy Group of the Institute of Modern Physics and Center of Mathematical Science of Zhejiang University in Hangzhou city, P.R.C. for the hospitality during the first stages of this work. The work of B.F. and R.H. is supported by Qiu-Shi Funding and the National Natural Science Foundation of China (NSFC) with Grant No.11135006, No.11125523 and No.11575156. R.H. would also like to acknowledge the supporting from Chinese Postdoctoral Administrative Committee. The work of C.C. is supported in part by the National Center for Theoretical Science (NCTS), Taiwan, Republic of China. The work of H.G. is supported by CNPq grant 403178/2014-2 and USC grant DGI-COCEIN-No 935-621115-N22.

\section{A A practical algorithm to determine the \pm sign in integration rules}

The systematic decomposition algorithm rewrites any CHY-integrand of higher-order poles as terms of simple poles, and the last step towards a final answer is to evaluate the resulting CHY-integrands by integration rules of simple poles. This requires a practical implementation of integration rules. In integration rules, the evaluation in fact contains two parts. The first is the contributing terms, which can be readily determined by working out the compatible combinations. The second is to determine the \pm sign of each contributing term, which is the topic we want to discuss here.

Again let us start from a generic $n$-point CHY-integrand (3.1), as (assuming here the canonical ordering, i.e., always $i<j$ ),

$$
\mathcal{I}=\frac{1}{\prod_{1 \leq i<j \leq n} z_{i j}^{\beta_{i j}}},
$$

respecting the Möbius invariance. Assuming that it has $m$ simple poles corresponding to $m$ subsets $A_{i}, i=1, \ldots, m$ of $\chi\left(A_{i}\right)=0$. Assuming also that one can construct $m^{\prime}$ compatible combinations $\left\{A_{\alpha_{1}}, A_{\alpha_{2}}, \ldots, A_{\alpha_{n-3}}\right\}$, etc., from $m$ subsets. Then by integration rule, the result is given by the summation of $m^{\prime}$ terms, with each term from a compatible combination as

$$
(-1)^{n+1+\mathcal{N}_{\text {inv }}} \frac{1}{s_{\alpha_{1}} s_{\alpha_{2}} \cdots s_{\alpha_{n-3}}},
$$

where $s_{\alpha_{i}}=\left(P_{A_{\alpha_{i}}}\right)^{2}$, and $\mathcal{N}_{\text {inv }}$ is the so-called inversion factor ${ }^{8}$ [31]. In the original derivation, the inversion factor is defined under a given gauge-fixing. One need to pick up a specific gauge and then consider what the inversion factor result. Briefly speaking, once a gauge is fixed, the inversion factor $n_{\text {inv }}$ of a subset $A_{\alpha}$ (avoiding the infinite fixed point) is the number of factor $z_{i j}$ with $i \in A_{\alpha}$ and $j \notin A_{\alpha}$, and $\mathcal{N}_{\text {inv }}$ is the sum of all $n_{\text {inv }}$ in a compatible combination. However, the choice of gauge makes it difficult to automatically compute the inversion factor for any given compatible combination.

\footnotetext{
${ }^{8}$ We thank Christian Baadsgaard for explaining the inversion factor to us.
} 
From the practices, we found that it is not necessary to restrict to a given gauge-fixing in order to compute the sign. Although the inversion factor is not the same in different gauges, the parity of $\mathcal{N}_{\text {inv }}$ is invariant, while the $( \pm)$ sign depends only on the parity. In fact, we can define the inversion factor without referring to any fixed points, as will be shown in the following.

For a length- $n$ set $\Sigma=\left\{\sigma_{1}, \sigma_{2}, \ldots, \sigma_{n}\right\}$, which is a permutation of $\{1,2, \ldots, n\}$, the signature of set $\Sigma$ is defined to be $(-1)^{\mathcal{N}}$, where $\mathcal{N}$ is the number of times required to iteratively permute two adjacent elements in order to arrive at the canonical ordering $\{1,2, \ldots, n\}$. For example,

$$
\{1,3,2,5,4,6\} \stackrel{2 \leftrightarrow 3}{\longrightarrow}\{1,2,3,5,4,6\} \quad \stackrel{4 \leftrightarrow 5}{\longrightarrow}\{1,2,3,4,5,6\},
$$

so $\mathcal{N}=2$. Of course there are many different permutations to do so, but the signature is invariant. Here we define the weighted signature $(-1)^{\mathcal{N}^{\prime}}$, where $\mathcal{N}^{\prime}$ is the weighted number of times. For each permutation of two adjacent elements $i \leftrightarrow j$, we count the number as $\beta_{i j}$ but not 1 , where $\beta_{i j}$ is determined by a given CHY-integrand. So for the above example, we have $\mathcal{N}^{\prime}=\beta_{23}+\beta_{45}$, and the weighted signature is $(-1)^{\beta_{23}+\beta_{45}}$.

Now we define the inversion factor $^{9}$ for a compatible combination $A=$ $\left\{A_{\alpha_{1}}, A_{\alpha_{2}}, \ldots, A_{\alpha_{n-3}}\right\}$ of a given CHY-integrand $\mathcal{I}\left(z_{i j}\right)$. Define the length- $n$ set $\Sigma=$ $\left\{\sigma_{1}, \sigma_{2}, \ldots, \sigma_{n}\right\}$ to be cyclically ordered, i.e., $\sigma_{n}$ and $\sigma_{1}$ are also considered to be adjacent. An adjacent subset $A_{\alpha}$ of $\Sigma$ is defined to be a subset of $\Sigma$ whose elements are adjacent in $\Sigma$ (but the ordering of elements in $A_{\alpha}$ dose not need to respect the ordering in $\Sigma$ ). For example, both $\left\{\sigma_{2}, \sigma_{3}, \sigma_{4}\right\}$ and $\left\{\sigma_{2}, \sigma_{4}, \sigma_{3}\right\}$ are adjacent subsets of $\Sigma$, while $\left\{\sigma_{1}, \sigma_{2}, \sigma_{n}\right\}$ is also an adjacent subset, but $\left\{\sigma_{2}, \sigma_{3}, \sigma_{5}\right\}$ is not.

A cyclically ordered length- $n$ set $\Sigma=\left\{\sigma_{1}, \sigma_{2}, \ldots, \sigma_{n}\right\}$ is said to be the parent set of a compatible combination $\left\{A_{\alpha_{1}}, A_{\alpha_{2}}, \ldots, A_{\alpha_{n-3}}\right\}$ if all subsets $A_{\alpha_{i}}$ 's are adjacent subsets of $\Sigma$. Provided we have already found a parent set $\Sigma$ of a compatible combination $A$, then the inversion factor $\mathcal{N}_{\mathrm{inv}}$ of $A$ is defined to be the weighted number $\mathcal{N}^{\prime}$ for permuting $\Sigma=\left\{\sigma_{1}, \sigma_{2}, \ldots, \sigma_{n}\right\}$ to canonical ordering $\{1,2, \ldots, n\}$, and the sign of the term associated to the compatible combination $A$ is nothing but $(-1)^{n+1+\mathcal{N}_{\text {inv }}}=(-1)^{n+1+\mathcal{N}^{\prime}}$, proportional to the weighted signature of parent set $\Sigma$. Of course, there will be more than one cyclically ordered sets which could be the parent set of a given compatible combination. Although the weighted number $\mathcal{N}^{\prime}$ of them are different, the parity of $\mathcal{N}^{\prime}$ is invariant, so is the weighted signature. In this case, we only need to find one parent set for a compatible combination, and compute the $( \pm)$ sign with it.

For a given compatible combination $A=\left\{A_{\alpha_{1}}, A_{\alpha_{2}}, \ldots, A_{\alpha_{n-3}}\right\}$ of CHY-integrand $\mathcal{I}\left(z_{i j}\right)$, now we shall find its parent set. Naively, one can generate all the permutation sets of $\{1,2, \ldots, n\}$ in Mathematica, and select one set which is the parent set of $A$. However, the number of permutation sets grows factorially with $n$, so it is practically not efficient. We propose the following strategy to construct a parent set of $A$. Remind that any two subsets in a compatible combination satisfy compatible condition, i.e., they should

\footnotetext{
${ }^{9}$ Note again here without referring to any gauge, which is different from the definition in [31].
} 
be nested $\left(A_{\alpha_{i}} \cup A_{\alpha_{j}}=A_{\alpha_{i}}\right.$ or $\left.A_{\alpha_{j}}\right)$ or disjoint $\left(A_{\alpha_{i}} \cap A_{\alpha_{j}}=\emptyset\right)$. The strategy starts from $B=\left\{A_{\alpha_{1}}\right\}$,

- If $A_{\alpha_{2}}$ is disjoint with $A_{\alpha_{1}}$, it will be included to get $B=\left\{A_{\alpha_{1}}, A_{\alpha_{2}}\right\}$,

- If $A_{\alpha_{2}}$ is nested with $A_{\alpha_{1}}$, we will replace $A_{\alpha_{1}}$ with a new subset $A_{\alpha_{1}}^{\prime}$, and consequently $B=\left\{A_{\alpha_{1}}^{\prime}\right\}$.

- In the case that $A_{\alpha_{1}} \subset A_{\alpha_{2}}$, we can define $A_{\alpha_{1}}^{\prime}=A_{\alpha_{1}}+\mathcal{C}\left[A_{\alpha_{2}}, A_{\alpha_{1}}\right]{ }^{10}$ where $\mathcal{C}\left[A_{\alpha_{i}}, A_{\alpha_{j}}\right]$ denotes the complement set of $A_{\alpha_{j}}$ with respective to $A_{\alpha_{i}}$, i.e., a set whose elements are in $A_{\alpha_{i}}$ but not $A_{\alpha_{j}}$. This construction is to ensure that $A_{\alpha_{1}}$ as well as $A_{\alpha_{2}}$ are the adjacent subsets of a parent set.

- In the case $A_{\alpha_{2}} \subset A_{\alpha_{1}}$, we can define $A_{\alpha_{1}}^{\prime}=A_{\alpha_{2}}+\mathcal{C}\left[A_{\alpha_{1}}, A_{\alpha_{2}}\right]$.

After $A_{\alpha_{2}}$ is done, we continue to $A_{\alpha_{3}}$, where now we should consider $A_{\alpha_{3}}$ to be nested or disjoint with all subsets in $B$ from previous step. For example, if in previous step we get $B=\left\{A_{\alpha_{1}}, A_{\alpha_{2}}\right\}$, then

- If $A_{\alpha_{3}}$ is disjoint with both $A_{\alpha_{1}}, A_{\alpha_{2}}$ (any subsets in $B$ ), we renew the set as $B=$ $\left\{A_{\alpha_{1}}, A_{\alpha_{2}}, A_{\alpha_{3}}\right\}$

- If $A_{\alpha_{3}} \subset A_{\alpha_{i}}$ (here $i=1$ or 2. Because from the construction, the subsets included in $B$ are always disjoint with each other, so $A_{\alpha_{3}}$ could only be a subset of either $A_{\alpha_{1}}$ or $A_{\alpha_{2}}$ ), we define $A_{\alpha_{i}}^{\prime}=A_{\alpha_{3}}+\mathcal{C}\left[A_{\alpha_{i}}, A_{\alpha_{3}}\right]$, and renew the set as $B=\left\{A_{\alpha_{1}}^{\prime}, A_{\alpha_{2}}\right\}$ or $B=\left\{A_{\alpha_{1}}, A_{\alpha_{2}}^{\prime}\right\}$,

- If any subsets in $B$ are subsets of $A_{\alpha_{3}}$,

- If only one subset in $B$ is a subset of the one under consideration, explicitly here $A_{\alpha_{i}} \subset A_{\alpha_{3}}(i=1$ or 2$)$, we define $A_{\alpha_{i}}^{\prime}=A_{\alpha_{i}}+\mathcal{C}\left[A_{\alpha_{3}}, A_{\alpha_{i}}\right]$, and renew the set as $B=\left\{A_{\alpha_{1}}^{\prime}, A_{\alpha_{2}}\right\}$ or $B=\left\{A_{\alpha_{1}}, A_{\alpha_{2}}^{\prime}\right\}$,

- If more than one subset in $B$ are subsets of the one under consideration, explicitly here $A_{\alpha_{1}} \subset A_{\alpha_{3}}$ and $A_{\alpha_{2}} \subset A_{\alpha_{3}}$, since $A_{\alpha_{1}}$ and $A_{\alpha_{2}}$ are disjoint, we can define a new subset

$$
A_{\alpha_{1}}^{\prime}=A_{\alpha_{1}}+A_{\alpha_{2}}+\mathcal{C}\left[A_{\alpha_{3}}, A_{\alpha_{1}}+A_{\alpha_{2}}\right]
$$

to replace $A_{\alpha_{1}}, A_{\alpha_{2}}$, and renew the set as $B=\left\{A_{\alpha_{1}}^{\prime}\right\}$.

With such strategy, we can enlarge set $B$ until the last $A_{\alpha_{n-3}}$ in the compatible combination is considered. The subsets in $B$ are by construction disjoint to each other. Assuming $B=\left\{B_{1}, B_{2}, \ldots, B_{k}\right\}$, a parent set of compatible combination $A$ can then be given by

$$
\Sigma=B_{1}+B_{2}+\cdots+B_{k}+\mathcal{C}\left[\{1,2, \ldots, n\}, \bigcup_{i=1}^{k} B_{k}\right] .
$$

\footnotetext{
${ }^{10} A_{1}+A_{2}$ stands for a new set with elements from $A_{1}$ followed by elements from $A_{2}$, keeping the ordering.
} 
The construction of parent set is already done, but we can go a step further. Because of the cyclic invariance, we can always fix 1 in the first position of $\Sigma$ as the convention. All above operations can be easily implemented in MATHEMATiCA.

It is better to understand above algorithm with an example. Let us consider the following six-point CHY-integrand with non-trivial numerator

$$
\mathcal{I}=\frac{z_{14} z_{35}}{z_{12} z_{13}^{2} z_{15} z_{16} z_{23} z_{24} z_{26} z_{34}^{2} z_{45}^{2} z_{56}^{2}},
$$

where explicitly, we have

$$
\begin{array}{llllll}
\beta_{12}=1, & \beta_{13}=2, & \beta_{14}=-1, & \beta_{15}=1, & \beta_{16}=1, & \beta_{23}=1, \\
\beta_{24}=1, & \beta_{26}=1, & \beta_{34}=2, & \beta_{35}=-1, & \beta_{45}=2, & \beta_{56}=2,
\end{array}
$$

and all others zero. It has six subsets of simple poles $\{1,3\},\{3,4\},\{4,5\},\{5,6\},\{1,2,3\}$, $\{1,5,6\}$, and from them we can construct three compatible combinations as

$$
\mathcal{B}_{1} \equiv\{\{1,3\},\{4,5\},\{1,2,3\}\}, \quad \mathcal{B}_{2} \equiv\{\{1,3\},\{5,6\},\{1,2,3\}\}, \quad \mathcal{B}_{3} \equiv\{\{3,4\},\{5,6\},\{1,5,6\}\} .
$$

Following the strategy, we have

$$
\begin{aligned}
& \mathcal{B}_{1}:\{\{1,3\}\} \stackrel{\text { consider }\{4,5\}}{\longrightarrow}\{\{1,3\},\{4,5\}\} \stackrel{\text { consider }\{1,2,3\}}{\longrightarrow}\{\{1,3,2\},\{4,5\}\}, \\
& \mathcal{B}_{2}:\{\{1,3\}\} \stackrel{\text { consider }\{5,6\}}{\longrightarrow}\{\{1,3\},\{5,6\}\} \stackrel{\text { consider }\{1,2,3\}}{\longrightarrow}\{\{1,3,2\},\{5,6\}\}, \\
& \mathcal{B}_{3}:\{\{3,4\}\} \stackrel{\text { consider }\{5,6\}}{\longrightarrow}\{\{3,4\},\{5,6\}\} \stackrel{\text { consider }\{1,5,6\}}{\longrightarrow}\{\{3,4\},\{5,6,1\}\} .
\end{aligned}
$$

So we can construct the parent set $\{1,3,2,4,5,6\}$ for $\{\{1,3\},\{4,5\},\{1,2,3\}\}$, parent set $\{1,3,2,5,6,4\}$ for $\{\{1,3\},\{5,6\},\{1,2,3\}\}$, and $\{3,4,5,6,1,2\}=\{1,2,3,4,5,6\}$ for $\{\{3,4\},\{5,6\},\{1,5,6\}\}$. Since

$$
\begin{array}{ll}
\{1,3,2,4,5,6\} & \stackrel{2 \leftrightarrow 3}{\longrightarrow}\{1,2,3,4,5,6\}, \\
\{1,3,2,5,6,4\} & \stackrel{2 \leftrightarrow 3}{\longrightarrow}\{1,2,3,5,6,4\} \quad \stackrel{4 \leftrightarrow 6}{\longrightarrow}\{1,2,3,5,4,6\} \quad \stackrel{4 \leftrightarrow 5}{\longrightarrow}\{1,2,3,4,5,6\},
\end{array}
$$

and the last one is already in canonical order, we have

$$
\mathcal{N}_{\mathrm{inv}}^{[1]}=\beta_{23}=1, \quad \mathcal{N}_{\mathrm{inv}}^{[2]}=\beta_{23}+\beta_{46}+\beta_{45}=3, \quad \mathcal{N}_{\mathrm{inv}}^{[3]}=0 .
$$

The sign of each term is given by $(-1)^{6+1+\mathcal{N}_{\text {inv }}^{[i]}}$, so we get the correct result

$$
\frac{1}{s_{13} s_{45} s_{123}}+\frac{1}{s_{13} s_{56} s_{123}}-\frac{1}{s_{34} s_{56} s_{156}} .
$$

Before end, let us present an example of the parity invariance of inversion factor. Consider again the afore-mentioned example and the compatible combination $\{\{1,3\},\{4,5\},\{1,2,3\}\}$. Among the $6 !=720$ permutation sets of $\{1,2,3,4,5,6\}$, there 
are 96 sets which could be the parent set of $A$. Restricting to the convention that 1 is always placed in the first position, we still get 16 parent sets as

$$
\begin{array}{llll}
\{1,2,4,6,5,3\}, & \{1,2,5,4,6,3\}, & \{1,2,5,6,4,3\}, & \{1,2,6,4,5,3\}, \\
\{1,3,2,4,6,5\}, & \{1,3,2,5,4,6\}, & \{1,3,2,5,6,4\}, & \{1,3,2,6,4,5\}, \\
\{1,3,4,6,5,2\}, & \{1,3,5,4,6,2\}, & \{1,3,5,6,4,2\}, & \{1,3,6,4,5,2\}, \\
\{1,4,6,5,2,3\}, & \{1,5,4,6,2,3\}, & \{1,5,6,4,2,3\}, & \{1,6,4,5,2,3\} .
\end{array}
$$

The inversion factors $\mathcal{N}_{\text {inv }}$ are consequently

$$
\mathcal{N}_{\text {inv }}=1,3,3,5,1,3,3,5,3,5,5,7,3,5,5,7
$$

which are all odd integers. Similarly, for compatible combination $\{\{3,4\},\{5,6\},\{1,5,6\}\}$, there are in total also 16 parent sets requiring 1 in the first position, as

$$
\begin{array}{llll}
\{1,2,3,4,5,6\}, & \{1,2,3,4,6,5\}, & \{1,2,4,3,5,6\}, & \{1,2,4,3,6,5\}, \\
\{1,3,4,2,5,6\}, & \{1,3,4,2,6,5\}, & \{1,4,3,2,5,6\}, & \{1,4,3,2,6,5\}, \\
\{1,5,6,2,3,4\}, & \{1,5,6,2,4,3\}, & \{1,5,6,3,4,2\}, & \{1,5,6,4,3,2\}, \\
\{1,6,5,2,3,4\}, & \{1,6,5,2,4,3\}, & \{1,6,5,3,4,2\}, & \{1,6,5,4,3,2\},
\end{array}
$$

and the inversion factors for them are respectively

$$
\mathcal{N}_{\text {inv }}=0,2,2,4,2,4,4,6,2,4,4,6,4,6,6,8
$$

which are all even integers. Thus the parity invariance of inversion factor is clearly shown. ${ }^{11}$

\section{B The identities from monodromy relation and cross-ratio identities}

In [30], an identity for generic pole $s_{12 \ldots k}$ is constructed from monodromy relations, as ${ }^{12}$

$$
\operatorname{Id}_{\{1, \ldots, k\}}=-\sum_{\sigma \in \widehat{\mathcal{O P}}(\{2, \ldots, k\},\{k+1, \ldots, n-1\})} \frac{P T\left(1, \sigma_{1}, \ldots, \sigma_{n-2}, n\right)}{P T(1, \ldots, n) s_{1 \cdots k}}\left(s_{1 \ldots k}+\sum_{\{i, j\} \mid \sigma_{i}>\sigma_{j}} s_{\sigma_{i} \sigma_{j}}\right),
$$

where $\widehat{\mathcal{O P}}(A, B)$ denotes the sets from ordered permutation of two sets $A, B$, i.e., all permutations among $A, B$ while keeping the ordering of $A$ and $B$ respectively, excluding the trivial one $\{2,3, \ldots, n-1\} . P T(1,2, \ldots, n)$ denotes the Parke-Taylor-like factor

$$
P T(1,2, \ldots, n)=\frac{1}{z_{12} z_{23} \cdots z_{n-1, n} z_{n 1}} .
$$

The fact that similar monodromy relations exist in CHY-integrands as those in string and gauge theory amplitudes is itself very interesting, while practically the identities

\footnotetext{
${ }^{11}$ Although this is confirmed by numerous computations, we should remark that there is not yet a proof on it.

${ }^{12} \mathrm{~A}$ derivation of the relation can be found in [32].
} 
from monodromy relations can be applied to the decomposition of CHY-integrands with higher-order poles. In fact, the systematic decomposition algorithm proposed in section 3 can as well proceed with the identities of monodromy relations as input, without any practical modifications. Here we shall briefly compare the decomposition algorithm with these two kinds of identities.

As mentioned, in a $n$-point scattering system, for a generic pole $s_{i_{1} i_{2} \cdots i_{k}}$, there exists $k(n-k)$ cross-ratio identities. The cross-ratio identity is almost symmetric, invariant under permutations on $A \backslash\{j\}$ and $\bar{A} \backslash\{p\}$. While for the identity of monodromy relation (B.1), because of the ordered permutation $\widehat{\mathcal{O P}}(A, B)$, each ordering of $A, B$ would define an identity for pole $s_{i_{1} i_{2} \cdots i_{k}}$. So naively, one would expect $k !(n-k)$ ! different identities of monodromy relations for a pole $s_{i_{1} i_{2} \cdots i_{k}}$. However, as far as there are enough identities to choose, this will not affect much the efficiency of decomposition algorithm.

The most important point related to the efficiency of computation via decomposition algorithm is the number of terms in an identity. Especially for the CHY-integrands with large $n$ and large $\Upsilon$ order of poles, the number of resulting terms of simples poles is very sensitive to the number of terms in identities. Furthermore, the more terms in an identity, the more troubles we would meet, since the chance of producing CHY-integrands with other higher-order poles will increase. For $s_{i_{1} i_{2} \cdots i_{k}}$ pole in $n$-point scattering, the number of terms in the identity of monodromy relation (MR) is

$$
\#[\mathrm{MR}]=\frac{(n-2) !}{(k-1) !(n-k-1) !}-1,
$$

while the number of terms in the cross-ratio identity (CR) is

$$
\#[\mathrm{CR}]=(n-k-1)(k-1) .
$$

It can be seen that (B.3) grows factorially while (B.4) grows algebraically. This matters a lot in the decomposition algorithm. For example, below is a table listing the number of terms in the identity of pole $s_{i_{1} i_{2} \cdots i_{k}}$ when $n=16$,

\begin{tabular}{|c|c|c|c|c|c|c|c|}
\hline$k$ & 2 & 3 & 4 & 5 & 6 & 7 & 8 \\
\hline$\#[\mathrm{MR}]$ & 13 & 90 & 363 & 1000 & 2001 & 3002 & 3431 \\
\hline$\#[\mathrm{CR}]$ & 13 & 24 & 33 & 40 & 45 & 48 & 49 \\
\hline
\end{tabular}

Although for pole $s_{i_{1} i_{2}}$, there is no difference, when considering a CHY-integrand with higher-order pole $s_{i_{1} i_{2} \cdots i_{k}}$ for large $k$, the difference becomes dramatic. For example, let us consider a 16-point CHY-integrand

$$
\mathcal{I}_{16}=\frac{1}{z_{12}^{2} z_{23}^{2} z_{34}^{2} z_{45}^{2} z_{56}^{2} z_{67}^{2} z_{78}^{2} z_{9,10}^{2} z_{10,11}^{2} z_{11,12}^{2} z_{12,13}^{2} z_{13,14}^{2} z_{14,15}^{2} z_{15,16}^{2} z_{18} z_{89} z_{9,16} z_{1,16}},
$$

with its 4-regular graph as shown in figure 6 . It has a double pole $s_{12345678}$, and a multiplication of cross-ratio identity

$$
\mathcal{I}_{16}=\mathcal{I}_{16} \times \mathbb{I}_{16}[\{1,2,3,4,5,6,7,8\}, 1,9]
$$

instantly produces 49 terms with simple poles. While for identity of monodromy relations the decomposition is much more difficult, leading to 3431 terms. 


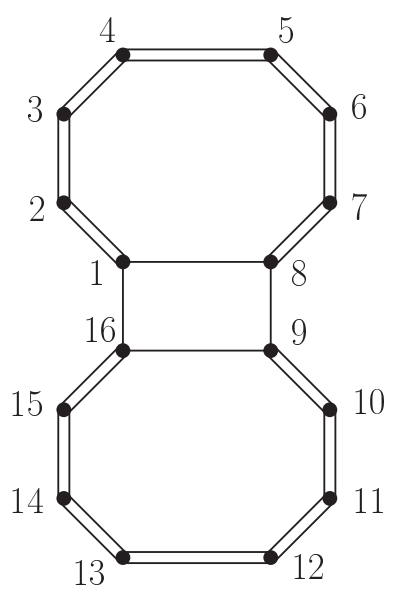

Figure 6. The 4-regular graph of a 16-point CHY-integrand.

C (Parke - Taylor $) \times($ Parke - Taylor $)$ geometry and its recurrence relation

In this section, let us consider the basic two-cycle CHY-integrands given by

$$
(\text { Parke }- \text { Taylor }) \times(\text { Parke }- \text { Taylor })=\mathrm{PT}^{2},
$$

i.e., graphs such as the following examples,
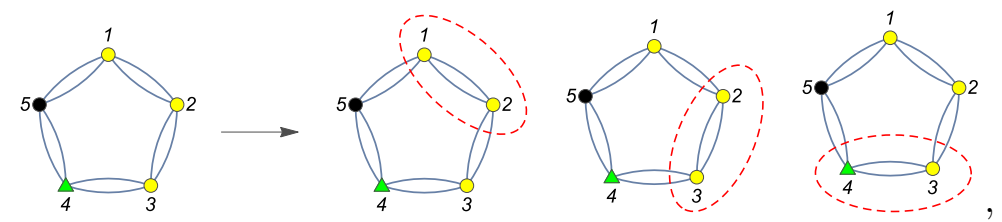

and
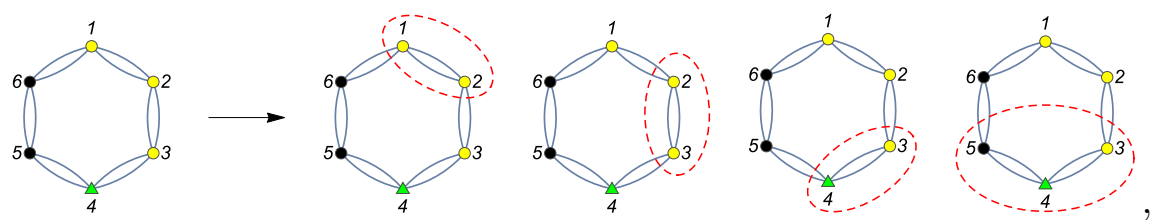

and
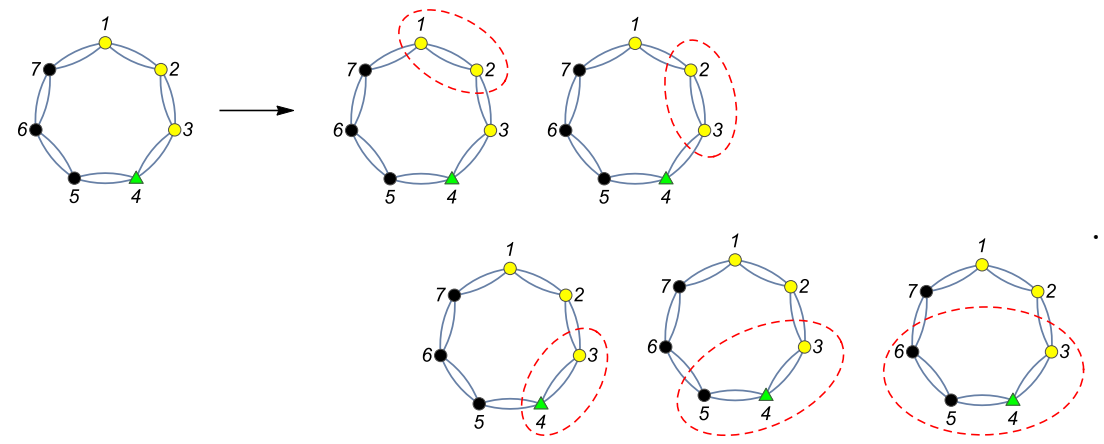

Although they can be computed trivially by integration rules of simple poles, let us repeat here by the means of recurrence relations of $\Lambda$-algorithm, with similar idea that can be 
generalized to other recurrence relations of more complicated geometry. Clearly, the number of all possible non-zero allowable configurations is $(n-2)$. From the $\Lambda$-algorithm, it is very well-known that each cut splits the original graph into two sub-graphs, which are of the same type, i.e., (Parke - Taylor) $)^{2}$.

Before writing this new recurrence relation, we give some definitions which will be useful. Let $\mathbb{\Phi}_{i}$ be the set of ordered elements given by

$$
\mathbb{D}_{i}:=\{5,6,7, \ldots i+4\}, \text { where } i \in\{1, \ldots, n-5\}, \quad n>5
$$

and we defined $\mathbb{D}_{0}=\emptyset$. Note that $\mathbb{D}_{1}=\{5\}, \mathbb{D}_{2}=\{5,6\}$ and so on. We also denote $\overline{\mathbb{D}}_{i}$ as the ordered complement of $\mathbb{D}_{i}$

$$
\overline{\mathbb{D}}_{i}:=\{5,6,7, \ldots, n-1\} \backslash \mathbb{D}_{i},
$$

for example

$$
\bar{\Phi}_{0}=\{5, \ldots, n-1\}, \quad \bar{\Phi}_{1}=\{6, \ldots, n-1\}, \quad \ldots, \quad \bar{\Phi}_{n-5}=\emptyset .
$$

With these definitions, the recurrence relation ${ }^{13}$ takes the form,

$$
\begin{aligned}
\mathcal{I}_{n}^{\mathrm{PT}^{2}}(1,2, \ldots, n)= & \frac{\mathcal{I}_{n-1}^{\mathrm{PT}^{2}}([1,2], 3, \ldots, n)}{\widetilde{s}_{34 \cdots n}}+\frac{\mathcal{I}_{n-1}^{\mathrm{PT}^{2}}(1,[2,3], \ldots, n)}{\widetilde{s}_{4 \cdots n 1}} \\
& +\sum_{i=0}^{n-5} \frac{\mathcal{I}_{n-i-1}^{\mathrm{PT}}\left(1,2,\left[3,4, \mathbb{\Phi}_{i}\right], \bar{\Phi}_{i}, n\right) \times \mathcal{I}_{i+3}^{\mathrm{PT}^{2}}\left(\left[\bar{\Phi}_{i}, n, 1,2\right], 3,4, \mathbb{\Phi}_{i}\right)}{\widetilde{s}_{34 \Phi_{i}}}
\end{aligned}
$$

where

$$
\mathcal{I}_{3}^{\mathrm{PT}^{2}}(a, b, c)=1
$$

which is the 3 -point function. It is very important to note that

$$
\mathcal{I}_{4}^{\mathrm{PT}^{2}}(1,2,3,4)=B(4,2 \mid 1,3)
$$

Finally, from the CHY-integrands given in (C.1), (C.2), (C.3) and the recurrence relation in (C.7), it is simple to check the five, six and seven-point examples

$$
\begin{aligned}
& \mathcal{I}_{5}^{\mathrm{PT}^{2}}(1,2,3,4,5)=\frac{\mathcal{I}_{4}^{\mathrm{PT}^{2}}([1,2], 3,4,5)}{\widetilde{s}_{345}}+\frac{\mathcal{I}_{4}^{\mathrm{PT}^{2}}(1,[2,3], 4,5)}{\widetilde{s}_{451}}+\frac{\mathcal{I}_{4}^{\mathrm{PT}^{2}}(1,2,[3,4], 5)}{\widetilde{s}_{34}}, \\
& \mathcal{I}_{6}^{\mathrm{PT}^{2}}(1,2,3,4,5,6)=\frac{\mathcal{I}_{5}^{\mathrm{PT}^{2}}([1,2], 3,4,5,6)}{\widetilde{s}_{3456}}+\frac{\mathcal{I}_{5}^{\mathrm{PT}^{2}}(1,[2,3], 4,5,6)}{\widetilde{s}_{4561}}+\frac{\mathcal{I}_{5}^{\mathrm{PT}^{2}}(1,2,[3,4], 5,6)}{\widetilde{s}_{34}} \\
& +\frac{\mathcal{I}_{4}^{\mathrm{PT}^{2}}(1,2,[3,4,5], 6) \times \mathcal{I}_{4}^{\mathrm{PT}^{2}}([6,1,2], 3,4,5)}{\widetilde{s}_{345}},
\end{aligned}
$$

\footnotetext{
${ }^{13}$ Let us remember that the $\Lambda$-algorithm is an iterative process.
} 
and

$$
\begin{aligned}
& \mathcal{I}_{7}^{\mathrm{PT}^{2}}(1,2,3,4,5,6,7)=\frac{\mathcal{I}_{6}^{\mathrm{PT}^{2}}([1,2], 3,4,5,6,7)}{\widetilde{s}_{34567}}+\frac{\mathcal{I}_{6}^{\mathrm{PT}^{2}}(1,[2,3], 4,5,6,7)}{\widetilde{s}_{45671}} \\
& +\frac{\mathcal{I}_{6}^{\mathrm{PT}^{2}}(1,2,[3,4], 5,6,7)}{\widetilde{s}_{34}} \\
& +\frac{\mathcal{I}_{5}^{\mathrm{PT}^{2}}(1,2,[3,4,5], 6,7) \times \mathcal{I}_{4}^{\mathrm{PT}^{2}}([6,7,1,2], 3,4,5)}{\widetilde{s}_{345}} \\
& +\frac{\mathcal{I}_{4}^{\mathrm{PT}^{2}}(1,2,[3,4,5,6], 7) \times \mathcal{I}_{5}^{\mathrm{PT}^{2}}([7,1,2], 3,4,5,6)}{\widetilde{s}_{3456}} .
\end{aligned}
$$

\section{Recurrence relation for $\mathcal{I}_{3,3}^{\mathrm{PT}^{2} \oplus \mathrm{PT}^{2}}, \mathcal{I}_{4,3}^{\mathrm{PT}^{2} \oplus \mathrm{PT}^{2}}, \mathcal{I}_{5,3}^{\mathrm{PT}^{2} \oplus \mathrm{PT}^{2}}$ and $\mathcal{I}_{6,3}^{\mathrm{PT}^{2} \oplus \mathrm{PT}^{2}}$}

In this section, we provide the results for the CHY-integrands

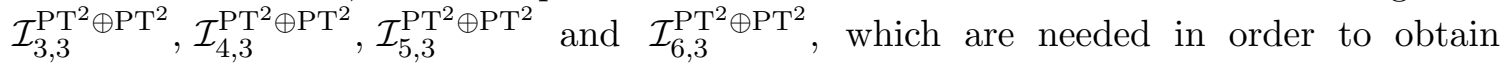
the final expression of (4.18). Applying the recurrence relation given in (4.17) one obtains

$$
\begin{aligned}
& \mathcal{I}_{3,3}^{\mathrm{PT}^{2} \oplus \mathrm{PT}^{2}}\left(\sigma_{1}, \sigma_{2}, \sigma_{3} \mid \sigma_{4}, \sigma_{5}, \sigma_{6}\right)=\frac{\mathcal{I}_{2,3}^{\mathrm{PT}^{2} \oplus \mathrm{PT}^{2}}\left(\left[\sigma_{3}, \sigma_{1}\right], \sigma_{2} \mid \sigma_{4}, \sigma_{5}, \sigma_{6}\right)}{\widetilde{s}_{\sigma_{3} \sigma_{1}}} \\
& +\frac{\mathcal{I}_{2,3}^{\mathrm{PT}^{2} \oplus \mathrm{PT}^{2}}\left(\sigma_{1},\left[\sigma_{2}, \sigma_{3}\right] \mid \sigma_{4}, \sigma_{5}, \sigma_{6}\right)}{\widetilde{s}_{\sigma_{2} \sigma_{3}}} \\
& +\frac{\mathcal{I}_{2,3}^{\mathrm{PT}^{2} \oplus \mathrm{PT}^{2}}\left(\left[\sigma_{1}, \sigma_{2}\right], \sigma_{3} \mid \sigma_{4}, \sigma_{5}, \sigma_{6}\right)}{\widetilde{s}_{\sigma_{3} \sigma_{4} \sigma_{5} \sigma_{6}}},
\end{aligned}
$$

and

$$
\begin{aligned}
& \mathcal{I}_{4,3}^{\mathrm{PT}^{2} \oplus \mathrm{PT}^{2}}\left(\sigma_{1}, \sigma_{2}, \sigma_{3}, \sigma_{4} \mid \sigma_{5}, \sigma_{6}, \sigma_{7}\right)=\frac{\mathcal{I}_{2,3}^{\mathrm{PT}^{2} \oplus \mathrm{PT}^{2}}\left(\left[\sigma_{3}, \sigma_{4}, \sigma_{1}\right], \sigma_{2} \mid \sigma_{5}, \sigma_{6}, \sigma_{7}\right) \times \mathcal{I}_{4}^{\mathrm{PT}^{2}}\left(\sigma_{1},\left[\sigma_{2}, \sigma_{5}, \sigma_{6}, \sigma_{7}\right], \sigma_{3}, \sigma_{4}\right)}{\widetilde{s}_{\sigma_{3} \sigma_{4} \sigma_{1}}} \\
& +\frac{\mathcal{I}_{3,3}^{\mathrm{PT}^{2} \oplus \mathrm{PT}^{2}}\left(\sigma_{1},\left[\sigma_{2}, \sigma_{3}\right] \sigma_{4} \mid \sigma_{5}, \sigma_{6}, \sigma_{7}\right) \times \mathcal{I}_{3}^{\mathrm{PT}^{2}}\left(\left[\sigma_{1}, \sigma_{4}, \sigma_{5}, \sigma_{6}, \sigma_{7}\right], \sigma_{2}, \sigma_{3}\right)}{\widetilde{s}_{\sigma_{2} \sigma_{3}}} \\
& +\frac{\mathcal{I}_{2,3}^{\mathrm{PT}^{2} \oplus \mathrm{PT}^{2}}\left(\sigma_{1},\left[\sigma_{2}, \sigma_{3}, \sigma_{4}\right] \mid \sigma_{5}, \sigma_{6}, \sigma_{7}\right) \times \mathcal{I}_{4}^{\mathrm{PT}^{2}}\left(\left[\sigma_{1}, \sigma_{5}, \sigma_{6}, \sigma_{7}\right], \sigma_{2}, \sigma_{3}, \sigma_{4}\right)}{\widetilde{s}_{\sigma_{2} \sigma_{3} \sigma_{4}}} \\
& +\frac{\mathcal{I}_{2,3}^{\mathrm{PT}^{2} \oplus \mathrm{PT}^{2}}\left(\left[\sigma_{1}, \sigma_{2}, \sigma_{4}\right], \sigma_{3} \mid \sigma_{5}, \sigma_{6}, \sigma_{7}\right) \times \mathcal{I}_{4}^{\mathrm{PT}^{2}}\left(\sigma_{1}, \sigma_{2},\left[\sigma_{3}, \sigma_{5}, \sigma_{6}, \sigma_{7}\right], \sigma_{4}\right)}{\widetilde{s}_{\sigma_{3} \sigma_{5} \sigma_{6} \sigma_{7}}} \\
& +\frac{\mathcal{I}_{3,3}^{\mathrm{PT}^{2} \oplus \mathrm{PT}^{2}}\left(\left[\sigma_{1}, \sigma_{2}\right] \sigma_{3}, \sigma_{4} \mid \sigma_{5}, \sigma_{6}, \sigma_{7}\right) \times \mathcal{I}_{3}^{\mathrm{PT}^{2}}\left(\sigma_{1}, \sigma_{2},\left[\sigma_{3}, \sigma_{4}, \sigma_{5}, \sigma_{6}, \sigma_{7}\right]\right)}{\widetilde{s}_{\sigma_{3} \sigma_{4} \sigma_{5} \sigma_{6} \sigma_{7}}},
\end{aligned}
$$

as well as

$$
\begin{aligned}
& \mathcal{I}_{5,3}^{\mathrm{PT}^{2} \oplus \mathrm{PT}^{2}}\left(\sigma_{1}, \sigma_{2}, \sigma_{3}, \sigma_{4}, \sigma_{5} \mid \sigma_{6}, \sigma_{7}, \sigma_{8}\right) \\
& =\frac{\mathcal{I}_{2,3}^{\mathrm{PT}^{2} \oplus \mathrm{PT}^{2}}\left(\left[\sigma_{3}, \sigma_{4}, \sigma_{5}, \sigma_{1}\right], \sigma_{2} \mid \sigma_{6}, \sigma_{7}, \sigma_{8}\right) \times \mathcal{I}_{5}^{\mathrm{PT}^{2}}\left(\sigma_{1},\left[\sigma_{2}, \sigma_{6}, \sigma_{7}, \sigma_{8}\right], \sigma_{3}, \sigma_{4}, \sigma_{5}\right)}{\widetilde{s}_{\sigma_{3} \sigma_{4} \sigma_{5} \sigma_{1}}} \\
& +\frac{\mathcal{I}_{4,3}^{\mathrm{PT}^{2} \oplus \mathrm{PT}^{2}}\left(\sigma_{1},\left[\sigma_{2}, \sigma_{3}\right] \sigma_{4}, \sigma_{5} \mid \sigma_{6}, \sigma_{7}, \sigma_{8}\right) \times \mathcal{I}_{3}^{\mathrm{PT}^{2}}\left(\left[\sigma_{1}, \sigma_{4}, \sigma_{5}, \sigma_{6}, \sigma_{7}, \sigma_{8}\right], \sigma_{2}, \sigma_{3}\right)}{\widetilde{s}_{\sigma_{2} \sigma_{3}}} \\
& +\frac{\mathcal{I}_{3,3}^{\mathrm{PT}^{2} \oplus \mathrm{PT}^{2}}\left(\sigma_{1},\left[\sigma_{2}, \sigma_{3}, \sigma_{4}\right], \sigma_{5} \mid \sigma_{6}, \sigma_{7}, \sigma_{8}\right) \times \mathcal{I}_{4}^{\mathrm{PT}^{2}}\left(\left[\sigma_{1}, \sigma_{5}, \sigma_{6}, \sigma_{7}, \sigma_{8}\right], \sigma_{2}, \sigma_{3}, \sigma_{4}\right)}{\widetilde{s}_{\sigma_{2} \sigma_{3} \sigma_{4}}}
\end{aligned}
$$




$$
\begin{aligned}
& +\frac{\mathcal{I}_{2,3}^{\mathrm{PT}^{2} \oplus \mathrm{PT}^{2}}\left(\sigma_{1},\left[\sigma_{2}, \sigma_{3}, \sigma_{4}, \sigma_{5}\right] \mid \sigma_{6}, \sigma_{7}, \sigma_{8}\right) \times \mathcal{I}_{5}^{\mathrm{PT}^{2}}\left(\left[\sigma_{1}, \sigma_{6}, \sigma_{7}, \sigma_{8}\right], \sigma_{2}, \sigma_{3}, \sigma_{4}, \sigma_{5}\right)}{\widetilde{s}_{\sigma_{2} \sigma_{3} \sigma_{4} \sigma_{5}}} \\
& +\frac{\mathcal{I}_{2,3}^{\mathrm{PT}^{2} \oplus \mathrm{PT}^{2}}\left(\left[\sigma_{1}, \sigma_{2}, \sigma_{4}, \sigma_{5}\right], \sigma_{3} \mid \sigma_{6}, \sigma_{7}, \sigma_{8}\right) \times \mathcal{I}_{5}^{\mathrm{PT}^{2}}\left(\sigma_{1}, \sigma_{2},\left[\sigma_{3}, \sigma_{6}, \sigma_{7}, \sigma_{8}\right], \sigma_{4}, \sigma_{5}\right)}{\widetilde{s}_{\sigma_{3} \sigma_{6} \sigma_{7} \sigma_{8}}} \\
& +\frac{\mathcal{I}_{3,3}^{\mathrm{PT}^{2} \oplus \mathrm{PT}^{2}}\left(\left[\sigma_{1}, \sigma_{2}, \sigma_{5}\right], \sigma_{3}, \sigma_{4} \mid \sigma_{6}, \sigma_{7}, \sigma_{8}\right) \times \mathcal{I}_{4}^{\mathrm{PT}^{2}}\left(\sigma_{1}, \sigma_{2},\left[\sigma_{3}, \sigma_{4}, \sigma_{6}, \sigma_{7}, \sigma_{8}\right], \sigma_{5}\right)}{\widetilde{s}_{\sigma_{3} \sigma_{4} \sigma_{6} \sigma_{7} \sigma_{8}}} \\
& +\frac{\mathcal{I}_{4,3}^{\mathrm{PT}^{2} \oplus \mathrm{PT}^{2}}\left(\left[\sigma_{1}, \sigma_{2}\right], \sigma_{3}, \sigma_{4}, \sigma_{5} \mid \sigma_{6}, \sigma_{7}, \sigma_{8}\right) \times \mathcal{I}_{3}^{\mathrm{PT}^{2}}\left(\sigma_{1}, \sigma_{2},\left[\sigma_{3}, \sigma_{4}, \sigma_{5}, \sigma_{6}, \sigma_{7}, \sigma_{8}\right]\right)}{\widetilde{s}_{\sigma_{3} \sigma_{4} \sigma_{5} \sigma_{6} \sigma_{7} \sigma_{8}}},
\end{aligned}
$$

and

$$
\begin{aligned}
& \mathcal{I}_{6,3}^{\mathrm{PT}^{2} \oplus \mathrm{PT}^{2}}\left(\sigma_{1}, \sigma_{2}, \sigma_{3}, \sigma_{4}, \sigma_{5}, \sigma_{6} \mid \sigma_{7}, \sigma_{8}, \sigma_{9}\right) \\
& =\frac{\mathcal{I}_{2,3}^{\mathrm{PT}^{2} \oplus \mathrm{PT}^{2}}\left(\left[\sigma_{3}, \sigma_{4}, \sigma_{5}, \sigma_{6}, \sigma_{1}\right], 2 \mid \sigma_{7}, \sigma_{8}, \sigma_{9}\right) \times \mathcal{I}_{6}^{\mathrm{PT}^{2}}\left(\sigma_{1},\left[\sigma_{2}, \sigma_{7}, \sigma_{8}, \sigma_{9}\right], \sigma_{3}, \sigma_{4}, \sigma_{5}, \sigma_{6}\right)}{\widetilde{s}_{\sigma_{3} \sigma_{4} \sigma_{5} \sigma_{6} \sigma_{1}}} \\
& +\frac{\mathcal{I}_{5,3}^{\mathrm{PT}^{2} \oplus \mathrm{PT}^{2}}\left(\sigma_{1},\left[\sigma_{2}, \sigma_{3}\right], \sigma_{4}, \sigma_{5}, \sigma_{6} \mid \sigma_{7}, \sigma_{8}, \sigma_{9}\right) \times \mathcal{I}_{3}^{\mathrm{PT}^{2}}\left(\left[\sigma_{1}, \sigma_{4}, \sigma_{5}, \sigma_{6}, \sigma_{7}, \sigma_{8}, \sigma_{9}\right], \sigma_{2}, \sigma_{3}\right)}{\widetilde{s}_{\sigma_{2} \sigma_{3}}} \\
& +\frac{\mathcal{I}_{4,3}^{\mathrm{PT}^{2} \oplus \mathrm{PT}^{2}}\left(\sigma_{1},\left[\sigma_{2}, \sigma_{3}, \sigma_{4}\right], \sigma_{5}, \sigma_{6} \mid \sigma_{7}, \sigma_{8}, \sigma_{9}\right) \times \mathcal{I}_{4}^{\mathrm{PT}^{2}}\left(\left[\sigma_{1}, \sigma_{5}, \sigma_{6}, \sigma_{7}, \sigma_{8}, \sigma_{9}\right], \sigma_{2}, \sigma_{3}, \sigma_{4}\right)}{\widetilde{s}_{\sigma_{2} \sigma_{3} \sigma_{4}}} \\
& +\frac{\mathcal{I}_{3,3}^{\mathrm{PT}^{2} \oplus \mathrm{PT}^{2}}\left(\sigma_{1},\left[\sigma_{2}, \sigma_{3}, \sigma_{4}, \sigma_{5}\right], \sigma_{6} \mid \sigma_{7}, \sigma_{8}, \sigma_{9}\right) \times \mathcal{I}_{5}^{\mathrm{PT}^{2}}\left(\left[\sigma_{1}, \sigma_{6}, \sigma_{7}, \sigma_{8}, \sigma_{9}\right], \sigma_{2}, \sigma_{3}, \sigma_{4}, \sigma_{5}\right)}{\widetilde{s}_{\sigma_{2} \sigma_{3} \sigma_{4} \sigma_{5}}} \\
& +\frac{\mathcal{I}_{2,3}^{\mathrm{PT}^{2} \oplus \mathrm{PT}^{2}}\left(\sigma_{1},\left[\sigma_{2}, \sigma_{3}, \sigma_{4}, \sigma_{5}, \sigma_{6}\right] \mid \sigma_{7}, \sigma_{8}, \sigma_{9}\right) \times \mathcal{I}_{6}^{\mathrm{PT}^{2}}\left(\left[\sigma_{1}, \sigma_{7}, \sigma_{8}, \sigma_{9}\right], \sigma_{2}, \sigma_{3}, \sigma_{4}, \sigma_{5}, \sigma_{6}\right)}{\widetilde{s}_{\sigma_{2} \sigma_{3} \sigma_{4} \sigma_{5} \sigma_{6}}} \\
& +\frac{\mathcal{I}_{2,3}^{\mathrm{PT}^{2} \oplus \mathrm{PT}^{2}}\left(\left[\sigma_{1}, \sigma_{2}, \sigma_{4}, \sigma_{5}, \sigma_{6}\right], \sigma_{3} \mid \sigma_{7}, \sigma_{8}, \sigma_{9}\right) \times \mathcal{I}_{6}^{\mathrm{PT}^{2}}\left(\sigma_{1}, \sigma_{2},\left[\sigma_{3}, \sigma_{7}, \sigma_{8}, \sigma_{9}\right], \sigma_{4}, \sigma_{5}, \sigma_{6}\right)}{\widetilde{s}_{\sigma_{3} \sigma_{7} \sigma_{8} \sigma_{9}}} \\
& +\frac{\mathcal{I}_{3,3}^{\mathrm{PT}^{2} \oplus \mathrm{PT}^{2}}\left(\left[\sigma_{1}, \sigma_{2}, \sigma_{5}, \sigma_{6}\right], \sigma_{3}, \sigma_{4} \mid \sigma_{7}, \sigma_{8}, \sigma_{9}\right) \times \mathcal{I}_{5}^{\mathrm{PT}^{2}}\left(\sigma_{1}, \sigma_{2},\left[\sigma_{3}, \sigma_{4}, \sigma_{7}, \sigma_{8}, \sigma_{9}\right], \sigma_{5}, \sigma_{6}\right)}{\widetilde{s}_{\sigma_{3} \sigma_{4} \sigma_{7} \sigma_{8} \sigma_{9}}} \\
& +\frac{\mathcal{I}_{4,3}^{\mathrm{PT}^{2} \oplus \mathrm{PT}^{2}}\left(\left[\sigma_{1}, \sigma_{2}, \sigma_{6}\right], \sigma_{3}, \sigma_{4}, \sigma_{5} \mid \sigma_{7}, \sigma_{8}, \sigma_{9}\right) \times \mathcal{I}_{4}^{\mathrm{PT}^{2}}\left(\sigma_{1}, \sigma_{2},\left[\sigma_{3}, \sigma_{4}, \sigma_{5}, \sigma_{7}, \sigma_{8}, \sigma_{9}\right], \sigma_{6}\right)}{\widetilde{s}_{\sigma_{3} \sigma_{4} \sigma_{5} \sigma_{7} \sigma_{8} \sigma_{9}}} \\
& +\frac{\mathcal{I}_{5,3}^{\mathrm{PT}^{2} \oplus \mathrm{PT}^{2}}\left(\left[\sigma_{1}, \sigma_{2}\right], \sigma_{3}, \sigma_{4}, \sigma_{5}, \sigma_{6} \mid \sigma_{7}, \sigma_{8}, \sigma_{9}\right) \times \mathcal{I}_{3}^{\mathrm{PT}^{2}}\left(\sigma_{1}, \sigma_{2},\left[\sigma_{3}, \sigma_{4}, \sigma_{5}, \sigma_{6}, \sigma_{7}, \sigma_{8}, \sigma_{9}\right]\right)}{\widetilde{s}_{\sigma_{3} \sigma_{4} \sigma_{5} \sigma_{6} \sigma_{7} \sigma_{8} \sigma_{9}}} .
\end{aligned}
$$

These results are checked numerically.

Open Access. This article is distributed under the terms of the Creative Commons Attribution License (CC-BY 4.0), which permits any use, distribution and reproduction in any medium, provided the original author(s) and source are credited.

\section{References}

[1] F. Cachazo, S. He and E.Y. Yuan, Scattering equations and Kawai-Lewellen-Tye orthogonality, Phys. Rev. D 90 (2014) 065001 [arXiv:1306.6575] [INSPIRE].

[2] F. Cachazo, S. He and E.Y. Yuan, Scattering of massless particles in arbitrary dimensions, Phys. Rev. Lett. 113 (2014) 171601 [arXiv:1307.2199] [INSPIRE]. 
[3] F. Cachazo, S. He and E.Y. Yuan, Scattering of massless particles: scalars, gluons and gravitons, JHEP 07 (2014) 033 [arXiv:1309.0885] [INSPIRE].

[4] F. Cachazo, S. He and E.Y. Yuan, Einstein-Yang-Mills scattering amplitudes from scattering equations, JHEP 01 (2015) 121 [arXiv:1409.8256] [INSPIRE].

[5] F. Cachazo, S. He and E.Y. Yuan, Scattering equations and matrices: from Einstein to Yang-Mills, DBI and NLSM, JHEP 07 (2015) 149 [arXiv:1412.3479] [INSPIRE].

[6] L. Dolan and P. Goddard, The polynomial form of the scattering equations, JHEP 07 (2014) 029 [arXiv: 1402.7374] [INSPIRE].

[7] Y.-H. He, C. Matti and C. Sun, The scattering variety, JHEP 10 (2014) 135 [arXiv: 1403.6833] [INSPIRE].

[8] C. Kalousios, Massless scattering at special kinematics as Jacobi polynomials, J. Phys. A 47 (2014) 215402 [arXiv:1312.7743] [INSPIRE].

[9] S. Weinzierl, On the solutions of the scattering equations, JHEP 04 (2014) 092 [arXiv: 1402.2516] [INSPIRE].

[10] C.S. Lam, Permutation symmetry of the scattering equations, Phys. Rev. D 91 (2015) 045019 [arXiv: 1410.8184] [INSPIRE].

[11] Y.-J. Du, F. Teng and Y.-S. Wu, CHY formula and MHV amplitudes, JHEP 05 (2016) 086 [arXiv: 1603.08158] [INSPIRE].

[12] C. Kalousios, Scattering equations, generating functions and all massless five point tree amplitudes, JHEP 05 (2015) 054 [arXiv: 1502.07711] [INSPIRE].

[13] C. Cardona and C. Kalousios, Comments on the evaluation of massless scattering, JHEP 01 (2016) 178 [arXiv : 1509.08908] [INSPIRE].

[14] C. Cardona and C. Kalousios, Elimination and recursions in the scattering equations, Phys. Lett. B 756 (2016) 180 [arXiv:1511.05915] [INSPIRE].

[15] L. Dolan and P. Goddard, General solution of the scattering equations, arXiv:1511.09441 [INSPIRE].

[16] R. Huang, J. Rao, B. Feng and Y.-H. He, An algebraic approach to the scattering equations, JHEP 12 (2015) 056 [arXiv: 1509.04483] [INSPIRE].

[17] M. Søgaard and Y. Zhang, Scattering equations and global duality of residues, Phys. Rev. D 93 (2016) 105009 [arXiv: 1509.08897] [INSPIRE].

[18] J. Bosma, M. Søgaard and Y. Zhang, The polynomial form of the scattering equations is an H-basis, Phys. Rev. D 94 (2016) 041701 [arXiv: 1605.08431] [INSPIRE].

[19] M. Zlotnikov, Polynomial reduction and evaluation of tree- and loop-level CHY amplitudes, JHEP 08 (2016) 143 [arXiv: 1605.08758] [INSPIRE].

[20] F. Cachazo and H. Gomez, Computation of contour integrals on $\mathcal{M}_{0, n}$, JHEP 04 (2016) 108 [arXiv: 1505.03571] [INSPIRE].

[21] H. Gomez, $\Lambda$ scattering equations, JHEP 06 (2016) 101 [arXiv:1604.05373] [INSPIRE].

[22] C. Cardona and H. Gomez, Elliptic scattering equations, JHEP 06 (2016) 094 [arXiv: 1605.01446] [INSPIRE].

[23] C. Baadsgaard, N.E.J. Bjerrum-Bohr, J.L. Bourjaily and P.H. Damgaard, Integration rules for scattering equations, JHEP 09 (2015) 129 [arXiv: 1506.06137] [INSPIRE]. 
[24] C. Baadsgaard, N.E.J. Bjerrum-Bohr, J.L. Bourjaily and P.H. Damgaard, Scattering equations and Feynman diagrams, JHEP 09 (2015) 136 [arXiv:1507.00997] [INSPIRE].

[25] C.S. Lam and Y.-P. Yao, Role of Möbius constants and scattering functions in Cachazo-He-Yuan scalar amplitudes, Phys. Rev. D 93 (2016) 105004 [arXiv:1512.05387] [INSPIRE].

[26] C.S. Lam and Y.-P. Yao, Evaluation of the Cachazo-He-Yuan gauge amplitude, Phys. Rev. D 93 (2016) 105008 [arXiv: 1602.06419] [INSPIRE].

[27] C. Baadsgaard, N.E.J. Bjerrum-Bohr, J.L. Bourjaily, P.H. Damgaard and B. Feng, Integration rules for loop scattering equations, JHEP 11 (2015) 080 [arXiv:1508.03627] [INSPIRE].

[28] C.R. Mafra, Berends-Giele recursion for double-color-ordered amplitudes, JHEP 07 (2016) 080 [arXiv: 1603.09731] [INSPIRE].

[29] R. Huang, B. Feng, M.-X. Luo and C.-J. Zhu, Feynman rules of higher-order poles in CHY construction, JHEP 06 (2016) 013 [arXiv: 1604.07314] [INSPIRE].

[30] N.E.J. Bjerrum-Bohr, J.L. Bourjaily, P.H. Damgaard and B. Feng, Analytic representations of Yang-Mills amplitudes, arXiv:1605.06501 [INSPIRE].

[31] C. Baadsgaard, Amplitudes from sting theory and CHY formalism, Master's thesis, http://discoverycenter.nbi.ku.dk/teaching/thesis_page/, Copenhagen University, Copenhagen Denmark (2015).

[32] Y.-X. Chen, Y.-J. Du and B. Feng, A proof of the explicit minimal-basis expansion of tree amplitudes in gauge field theory, JHEP 02 (2011) 112 [arXiv:1101.0009] [INSPIRE]. 